\title{
Synchronization Control for Discrete-Time Delayed Dynamical Networks with Switching Topology under Actuator Saturations
}

\author{
Yonggang Chen, Zidong Wang, Jun $\mathrm{Hu}$ and Qing-Long Han
}

\begin{abstract}
This paper is concerned with the synchronization control problem for a class of discrete-time dynamical networks with mixed delays and switching topology. The saturation phenomenon of physical actuators is specifically considered in designing feedback controllers. By exploring the mixed-delay-dependent sector conditions in combination with the piecewise Lyapunovlike functional and the average-dwell-time switching, a sufficient condition is first established under which all trajectories of the error dynamics are bounded for admissible initial conditions and non-zero external disturbances, while the $l_{2}-l_{\infty}$ performance constraint is satisfied. Furthermore, the exponential stability of the error dynamics is ensured for admissible initial conditions in the absence of disturbances. Secondly, by using some congruence transformations, the explicit condition guaranteeing the existence of desired controller gains is obtained in terms of the feasibility of a set of linear matrix inequalities. Then, three convex optimization problems are formulated regarding the disturbance tolerance, the $l_{2}-l_{\infty}$ performance and the initial condition set, respectively. Finally, two simulation examples are given to show the effectiveness and merits of the proposed results.
\end{abstract}

Index Terms-Synchronization control, discrete-time networks, dynamical networks, mixed delays, switching topology, actuator saturations.

\section{INTRODUCTION}

Over the past several decades, the complex dynamical networks have received significant research attention due to their wide applications in social networks, transportation networks, electric power grids and neural networks, etc [1], [9], [16], [31], [38], [41], [43]. Moreover, it has been generally recognized that the synchronization problem among all dynamical nodes is one of the most interesting research issues

This work was supported in part by the National Natural Science Foundation of China under Grants 61773156, 61873148, 61673141 and 61933007, in part by the Program for Science and Technology Innovation Talents in the Universities of Henan Province of China under Grant 19HASTIT028, in part by the Research Fund for the Taishan Scholar Project of Shandong Province of China, in part by the Royal Society of the U.K., and in part the Alexander von Humboldt Foundation of Germany. (Corresponding author: Zidong Wang)

Y. Chen is with the School of Electrical Engineering and Automation, Henan Polytechnic University, Jiaozuo 454000, China, and is also with the School of Mathematical Sciences, Henan Institute of Science and Technology, Xinxiang 453003, China. (e-mail: happycygzmd@tom.com).

Z. Wang is with the College of Electrical Engineering and Automation, Shandong University of Science and Technology, Qingdao 266590, China, and is also with the Department of Computer Science, Brunel University London, Uxbridge UB8 3PH, U.K. (e-mail: Zidong.Wang@brunel.ac.uk).

$\mathrm{J}$. $\mathrm{Hu}$ is with the Department of Applied Mathematics, Harbin University of Science and Technology, Harbin 150080, China (e-mail: hujun2013@gmail.com).

Q.-L. Han is with the School of Software and Electrical Engineering, Swinburne University of Technology, Melbourne, VIC 3122, Australia (email: qhan@swin.edu.au). for complex dynamical networks [11], [28], [30], [36], [39], [40], [50], [58]. On the other hand, time-delays are frequently encountered in complex dynamical networks because of the parallel signal transmission among a large number of nodes as well as the complicated couplings of dynamical networks. As time-delays could lead to poor network performance or even undesirable instability, considerable research interest has been devoted to the synchronization problem of complex dynamical networks with various types of time-delays [13], [23], [27], [29], [44]. In particular, when a dynamical network cannot achieve the synchronization by itself or the network is expected to possess better performance, a variety of control techniques have been proposed during the past decade, see e.g. [2], [14], [21], [26], [35], [49], [56]. For example, an event-triggered approach has been adopted in [14] for discretetime dynamical networks with coupling delay, the pinning impulsive scheme has been proposed in [26] for a class of nonlinear dynamical networks with time-varying delay, and the adaptive control scheme has been employed in [56] for a general nonlinear dynamical network with delayed nodes.

Despite the extensive investigation on dynamical networks, the vast majority of existing results are concerned with dynamical networks with stationary/static topologies. In reality, however, the network topology may be time-varying due probably to the link failures, the mobility of networks and the creation of new links [34], [59]. To characterize the dynamical (sometimes even abrupt) changes of the topology structure, two types of network topologies have been introduced in the literature, one is the switching topology and the other is the Markov jump topology. Here, the switching topology means that the topology changes according to certain deterministic switching logic (including the arbitrary switching and the designed switching) [59]. In the past decade, several remarkable results have been obtained on the dynamical networks with switching topology or Markov jump topology [15], [17], [22], [24], [25], [45], [59]. For example, the synchronization problem has been addressed in [25] for delayed dynamical networks with switching topology under the average dwell time (ADT) switching. The impulsive synchronization scheme has been used in [15] to consider the discrete delayed stochastic complex networks with switching topology. In [45], the pinning synchronization problem has been studied for directed networks with switching topology by using multiple Lyapunov function approach.

In many feedback control systems, actuator saturation is an unavoidable phenomenon because of the inherent constrains on physical actuators. Actuator saturation constitutes yet another 


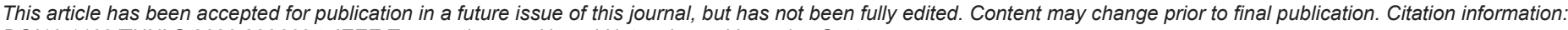
DOI10.1109/TNNLS.2020.2996094, IEEE Transactions on Neural Networks and Learning Systems

FINAL VERSION

source of instability and performance degradation. In the past two decades, the analysis and synthesis problems have been under extensive investigation for control systems subject to actuator saturations [12], [19], [20], [37], [42], [47], [51], [52]. It has been shown that the global results are almost impossible to be achieved if the open-loops of control systems are exponentially unstable [20], [47]. As such, the local/regional design has gained an ever-increasing interest [19], [37], [51], [52]. In general, two types of models have been developed to deal with the saturation nonlinearity, namely, the polytopic models and the models with generalized sector conditions. For systems with both time-delays and saturating actuators, the corresponding results have been established in [4], [6], [33], [57]. In particular, it is noted that the delay-dependent polytopic models have been proposed in [4], [6] in order to obtain some improved stabilization conditions.

In the past years, the synchronization control problem has been widely investigated for dynamical networks with actuator saturations [3], [7], [46], [48]. For example, the sampled-data control scheme has been used in [46] for nonlinear dynamical networks with actuator saturations. By utilizing the information from both time-delays and the nodes involving saturation nonlinearities, the exponential synchronization problem has been studied in [7] for delayed dynamical networks. Nevertheless, it is worth pointing out that the network topologies in existing literature have been assumed to be stationary/static and the external disturbances have been largely overlooked. Moreover, the existing results have been mainly concerned with the continuous-time dynamical networks without time-delays (or with a single discrete delay). Clearly, the synchronization control problem has not been examined yet for discretetime delayed dynamical networks with switching topology and actuator saturations, not to mention the case that the distributed delay and external disturbances are also involved. This situation has motivated our current investigation.

In this paper, we aim to address the synchronization control problem for a class of discrete-time dynamical networks with mixed delays, switching topology and actuator saturations. By using the combination of novel sector conditions, a piecewise Lyapunov-like functional and the ADT switching, a sufficient condition is first obtained under which the error dynamics can achieve some desirable performance indices including the boundedness, the $l_{2}-l_{\infty}$ performance and the exponential stability. Then, an explicit condition is obtained for designing desirable controllers by means of the feasibility of some linear matrix inequalities (LMIs). Subsequently, several optimization problems have been proposed to obtain the optimal performance indices. Finally, two simulation examples demonstrate the values of our obtained results. The main contributions of this paper are summarized as follows. 1) The synchronization control problem is addressed, for the first time, for dynamical networks with both switching topology and time delays under actuator saturations within a discrete-time framework. 2) For discrete-time dynamics with mixed delays, this paper proposes the mixed-delay-dependent sector conditions. Specially, the distributed-delay term $\sum_{l=1}^{+\infty} \nu_{l} \bar{h}(e(k-l))$ is introduced for the first time in our sector conditions. Moreover, different from the existing approaches using the augmented Lyapunov- like functionals to facilitate the utilization of delay-dependent sector conditions [7], the analysis procedure in this paper is based on a fairly simple yet effective Lyapunov-like functional that proves to be more appropriate for large-scale networks. 3) Different from the weighted $l_{2}-l_{\infty}$ performance index employed in [8], [10] for switched systems under the ADT switching, the $l_{2}-l_{\infty}$ performance proposed in this paper is in a non-weighted framework and is shown to be more effective for dynamical networks with switching topology.

Notation. The superscript " $T$ " refers to the matrix transposition. $\mathbb{R}^{n}$ and $\mathbb{R}^{n \times n}$ denote the $n$-dimensional Euclidean space and the set of all $n \times n$ real matrices, respectively. $\|x\|$ stands for the Euclidean norm of a vector $x$. The matrix $P>0$ $(P \geq 0)$ denotes that $P$ is real, symmetric and positive definite (positive semi-definite). $\lambda_{M}(P)$ and $\lambda_{m}(P)$ are the maximum and minimum eigenvalue of the matrix $P$, respectively. $u_{(l)}$ denotes the $l$-th element of a vector $u$ and $K_{(l)}$ stands for the $l$-th row of the matrix $K . I$ is an identity matrix with proper dimension and $\otimes$ denotes the Kronecker product. The symmetric terms in a symmetric matrix are denoted by $*$. Also, for vector functions $e(k)$ and $\bar{\omega}(k)(k \geq 0)$, we denote

$$
\|e(k)\|_{\infty} \triangleq \sup _{k \geq 0} \sqrt{e^{T}(k) e(k)},\|\bar{\omega}(k)\|_{2} \triangleq \sqrt{\sum_{k=0}^{+\infty} \bar{\omega}^{T}(k) \bar{\omega}(k) .}
$$

\section{PROBlem DESCRIPTION}

Consider the following class of discrete-time dynamical networks consisting of $N$ identical coupled nodes subject to mixed delays, switching topology and actuator saturations:

$$
\left\{\begin{array}{c}
x_{i}(k+1)=C x_{i}(k)+A f\left(x_{i}(k)\right)+D_{1} g\left(x_{i}\left(k-\tau_{k}\right)\right) \\
+D_{2} \sum_{l=1}^{+\infty} \nu_{l} h\left(x_{i}(k-l)\right)+\sum_{j=1}^{N} \tilde{l}_{i j}^{\sigma k} \Gamma x_{j}(k) \\
+B \operatorname{sat}\left(u_{i}(k)\right)+E \omega_{i}(k), \\
x_{i}(\theta)=\phi_{i}(\theta), \theta \in(-\infty, 0], i=1,2, \ldots, N
\end{array}\right.
$$

where $x_{i}(k) \in \mathbb{R}^{n}, u_{i}(k) \in \mathbb{R}^{m}, \omega_{i}(k) \in \mathbb{R}^{\varsigma}$ and $\phi_{i}(\theta) \in \mathbb{R}^{n}$ $(i \in\{1,2, \ldots, N\} \triangleq \mathcal{N})$ denote the state vector, the control input, the disturbance input and the initial condition of the $i$-th node, respectively. $A, B, C, D_{1}, D_{2}$ and $E$ are known constant matrices with appropriate dimensions. $\tau_{k}$ is the time-varying discrete delay satisfying $\underline{\tau} \leq \tau_{k} \leq \bar{\tau}$, where $\underline{\tau}$ and $\bar{\tau}$ are known non-negative integers. $\sigma_{k}$ is a piecewise constant function taking values in a finite set $\{1,2, \cdots, M\} \triangleq \mathcal{M}$, which is used to the characterize the switching behavior of the network topology. $\Gamma$ denote the inner-coupling matrix, and $L_{\alpha}=\left(\tilde{l}_{i j}^{\alpha}\right)_{N \times N}$ $(\alpha \in \mathcal{M})$ is a matrix representing the outer-coupling configuration with $\tilde{l}_{i j}^{\alpha} \geq 0(i \neq j)$ and $\sum_{j=1}^{N} \tilde{l}_{i j}^{\alpha}=0$. $\operatorname{sat}\left(u_{i}\right)=$ $\left[\operatorname{sat}\left(u_{i(1)}\right) \operatorname{sat}\left(u_{i(2)}\right) \cdots \operatorname{sat}\left(u_{i(m)}\right)\right]^{T}$ is a standard vectorvalued saturation function with the saturation levels $\bar{u}_{i(j)}>$ 0 , where $\operatorname{sat}\left(u_{i(j)}\right)=\operatorname{sgn}\left(u_{i(j)}\right) \min \left\{\left|u_{i(j)}\right|, \bar{u}_{i(j)}\right\} \quad(j=$ $1,2, \ldots, m)$. The term involving the sum $\sum_{l=1}^{+\infty} \nu_{l} h\left(x_{i}(k-l)\right)$ is referred to as the infinite distributed delay term. 
Throughout this paper, it is assumed that there exists a scalar $0<\rho<1$ such that the following condition holds:

$$
\sum_{l=1}^{+\infty} \nu_{l}<\sum_{l=1}^{+\infty} \nu_{l} \rho^{-l}<+\infty
$$

For vector-valued functions $f(x) \in \mathbb{R}^{n}, g(x) \in \mathbb{R}^{n}$ and $h(x) \in \mathbb{R}^{n}$, we assume that they are continuous with $f(0)=$ $g(0)=h(0)=0$ and satisfy the following conditions [23]:

$$
\left\{\begin{array}{l}
{\left[f(x)-f(y)-F_{1}(x-y)\right]^{T}} \\
\times\left[f(x)-f(y)-F_{2}(x-y)\right] \leq 0, \\
{\left[g(x)-g(y)-G_{1}(x-y)\right]^{T}} \\
\times\left[g(x)-g(y)-G_{2}(x-y)\right] \leq 0, \\
{\left[h(x)-h(y)-H_{1}(x-y)\right]^{T}} \\
\times\left[h(x)-h(y)-H_{2}(x-y)\right] \leq 0
\end{array}\right.
$$

where $x, y \in \mathbb{R}^{n}$, and $F_{1}, F_{2}, G_{1}, G_{2}, H_{1}, H_{2} \in \mathbb{R}^{n \times n}$ are known real constant matrices.

Remark 1: The conditions (3) are well-known sector-like descriptions of the nonlinearities. The nonlinearities in many systems, such as Lorenz system, Chua's circuit, Chen system and recurrent neural networks, satisfy the sector conditions (3). Compared with the usual Lipschitz assumption, it is seen that the sector-like assumption is more general. Of course, the conditions (3) are stricter than the semi-Lipschitz conditions [2], [50]. However, by using (3), one can obtain less conservative results since more information on nonlinearities is utilized.

In this paper, the isolate node has the following form:

$$
\left\{\begin{aligned}
& s(k+1)=C s(k)+A f(s(k))+D_{1} g\left(s\left(k-\tau_{k}\right)\right) \\
&+D_{2} \sum_{l=1}^{+\infty} \nu_{l} h(s(k-l))+E \omega(k), \\
& s(\theta)=\phi(\theta), \theta \in(-\infty, 0]
\end{aligned}\right.
$$

where $s(k) \in \mathbb{R}^{n}, \omega(k) \in \mathbb{R}^{\varsigma}$ and $\phi(\theta) \in \mathbb{R}^{n}$ are, respectively, the state vector, the disturbance input and the initial condition.

Let us introduce the following new notations:

$$
\begin{aligned}
& e_{i}(k) \triangleq x_{i}(k)-s(k), \bar{\omega}_{i}(k) \triangleq \omega_{i}(k)-\omega(k), \\
& \bar{\phi}_{i}(\theta) \triangleq \phi_{i}(\theta)-\phi(\theta), \bar{L}_{\sigma_{k}} \triangleq L_{\sigma_{k}} \otimes \Gamma \text {, } \\
& \bar{f}\left(e_{i}(k)\right) \triangleq f\left(x_{i}(k)\right)-f(s(k))-F_{1} e_{i}(k), \\
& \bar{g}\left(e_{i}(k)\right) \triangleq g\left(x_{i}(k)\right)-g(s(k))-G_{1} e_{i}(k), \\
& \bar{h}\left(e_{i}(k)\right) \triangleq h\left(x_{i}(k)\right)-h(s(k)), \bar{C} \triangleq I_{N} \otimes C, \\
& \bar{A} \triangleq I_{N} \otimes A, \bar{B} \triangleq I_{N} \otimes B, \bar{E} \triangleq I_{N} \otimes E, \\
& \bar{D}_{j} \triangleq I_{N} \otimes D_{j}, \quad \bar{F}_{j} \triangleq I_{N} \otimes F_{j}, j=1,2, \\
& \bar{G}_{j} \triangleq I_{N} \otimes G_{j}, \bar{H}_{j} \triangleq I_{N} \otimes H_{j}, j=1,2, \\
& e(k) \triangleq\left[\begin{array}{llll}
e_{1}^{T}(k) & e_{2}^{T}(k) & \cdots & \left.e_{N}^{T}(k)\right)
\end{array}\right]^{T}, \\
& \bar{f}(e(k)) \triangleq\left[\begin{array}{llll}
\bar{f}^{T}\left(e_{1}(k)\right) & \bar{f}^{T}\left(e_{2}(k)\right) & \cdots & \left.\bar{f}^{T}\left(e_{N}(k)\right)\right)
\end{array}\right]^{T}, \\
& \bar{g}(e(k)) \triangleq\left[\begin{array}{llll}
\bar{g}^{T}\left(e_{1}(k)\right) & \bar{g}^{T}\left(e_{2}(k)\right) & \cdots & \left.\bar{g}^{T}\left(e_{N}(k)\right)\right)
\end{array}\right]^{T}, \\
& \bar{h}(e(k)) \triangleq\left[\begin{array}{llll}
\bar{h}^{T}\left(e_{1}(k)\right) & \bar{h}^{T}\left(e_{2}(k)\right) & \cdots & \left.\bar{h}^{T}\left(e_{N}(k)\right)\right)
\end{array}\right]^{T}, \\
& \operatorname{sat}(u) \triangleq\left[\begin{array}{llll}
\operatorname{sat}^{T}\left(u_{1}\right) & \operatorname{sat}^{T}\left(u_{2}\right) & \cdots & \operatorname{sat}^{T}\left(u_{N}\right)
\end{array}\right]^{T}, \\
& \bar{\omega}(k) \triangleq\left[\begin{array}{llll}
\bar{\omega}_{1}^{T}(k) & \bar{\omega}_{2}^{T}(k) & \cdots & \left.\bar{\omega}_{N}^{T}(k)\right)
\end{array}\right]^{T}, \\
& \bar{\phi}(\theta) \triangleq\left[\begin{array}{llll}
\bar{\phi}_{1}^{T}(\theta) & \bar{\phi}_{2}^{T}(\theta) & \cdots & \bar{\phi}_{3}^{T}(\theta)
\end{array}\right]^{T} .
\end{aligned}
$$

Then, we have the following error dynamics:

$$
\left\{\begin{array}{l}
e(k+1)=\left(\bar{C}+\bar{F}_{1}+\bar{L}_{\sigma_{k}}\right) e(k)+\bar{G}_{1} e\left(k-\tau_{k}\right) \\
\quad+\bar{A} \bar{f}(e(k))+\bar{D}_{1} \bar{g}\left(e\left(k-\tau_{k}\right)\right)+\bar{D}_{2} \\
\quad \times \sum_{l=1}^{+\infty} \nu_{l} \bar{h}(e(k-l))+\bar{B} \operatorname{sat}(u(k))+\bar{E} \bar{\omega}(k), \\
e(\theta)=\bar{\phi}(\theta), \theta \in(-\infty, 0] .
\end{array}\right.
$$

Using the conditions in (3), we can see that

$$
\left\{\begin{array}{l}
\bar{f}^{T}(e(k))\left[\bar{f}(e(k))-\left(\bar{F}_{2}-\bar{F}_{1}\right) e(k)\right] \leq 0, \\
\bar{g}^{T}(e(k))\left[\bar{g}(e(k))-\left(\bar{G}_{2}-\bar{G}_{1}\right) e(k)\right] \leq 0, \\
{\left[\bar{h}(e(k))-\bar{H}_{1} e(k)\right]^{T}\left[\bar{h}(e(k))-\bar{H}_{2} e(k)\right] \leq 0}
\end{array}\right.
$$

In addition, we assume that the disturbance $\bar{\omega}(k)$ of (5) is energy-bounded and satisfy the following condition:

$$
\sum_{k=0}^{+\infty} \bar{\omega}^{T}(k) \bar{\omega}(k) \leq \delta
$$

where $\delta$ is known positive scalar.

Remark 2: It is noted that the component $\bar{\omega}_{i}(k)(i \in \mathcal{N})$ of $\bar{\omega}(k)$ in (5) is actually the disturbance error between the disturbance $\omega_{i}(k)$ in the network (1) and the disturbance $\omega(k)$ in the isolate node (4). Therefore, the condition (7) can be written as $\sum_{k=0}^{+\infty} \sum_{i=1}^{N}\left[\omega_{i}(k)-\omega(k)\right]^{T}\left[\omega_{i}(k)-\omega(k)\right] \leq \delta$. If the disturbance $\omega(k)$ in the isolate node (4) is absent, then the condition (7) becomes $\sum_{k=0}^{+\infty} \sum_{i=1}^{N} \omega_{i}(k)^{T} \omega_{i}(k) \leq \delta$.

In order to achieve the synchronization of the dynamical network (1), we employ the following switching controller:

$$
u_{i}(k)=K_{i}^{\sigma_{k}} e_{i}(k), i=1,2, \ldots, N
$$

where $\sigma_{k}$ is the switching signal denoted in the model (1) and $K_{i}^{\alpha} \in \mathbb{R}^{m \times n}(\alpha \in \mathcal{M})$ is the controller gain matrix.

Defining the function $\psi(z) \triangleq z-\operatorname{sat}(z)$ and using the controller (8), we obtain the following closed-loop dynamics:

$$
\left\{\begin{aligned}
e(k & +1)=\left(\bar{C}+\bar{F}_{1}+\bar{L}_{\sigma_{k}}+\bar{B} K_{\sigma_{k}}\right) e(k) \\
& +\bar{G}_{1} e\left(k-\tau_{k}\right)+\bar{A} \bar{f}(e(k))+\bar{D}_{1} \bar{g}\left(e\left(k-\tau_{k}\right)\right) \\
& +\bar{D}_{2} \sum_{l=1}^{+\infty} \nu_{l} \bar{h}(e(k-l))-B \psi(u(k))+\bar{E} \omega(k), \\
e(\theta) & =\bar{\phi}(\theta), \theta \in(-\infty, 0]
\end{aligned}\right.
$$

where

$$
\begin{aligned}
K_{\sigma_{k}} & \triangleq \operatorname{diag}\left\{K_{1}^{\sigma_{k}}, K_{2}^{\sigma_{k}}, \ldots, K_{N}^{\sigma_{k}}\right\}, \\
\psi(u(k)) & \triangleq\left[\psi^{T}\left(u_{1}(k)\right) \quad \psi^{T}\left(u_{2}(k)\right) \cdots \psi^{T}\left(u_{N}(k)\right)\right]^{T} .
\end{aligned}
$$

Next, we introduce the following definition and lemma.

Definition 1: [18] For any $k>l \geq k_{0}$, let $\mathbb{N}_{\sigma}(l, k)$ be the switching number of $\sigma$ over $[l, k]$. If $\mathbb{N}_{\sigma}(l, k) \leq \mathbb{N}_{0}+(k-$ l) $/ \mathbb{T}_{a}$ holds for any given $\mathbb{T}_{a}>0$ and $\mathbb{N}_{0} \geq 0$, then $\mathbb{T}_{a}$ and $\mathbb{N}_{0}$ are called ADT and chatter bound, respectively.

Lemma 1: [23], [32] Let $Z \in \mathbb{R}^{n \times n}$ be a positive definite matrix, $x_{i} \in \mathbb{R}^{n}$, and $a_{i} \geq 0, b_{i} \geq 0(i=1,2, \cdots)$. If the sequences concerned are convergent, then we have

$$
\left(\sum_{i=1}^{+\infty} a_{i} x_{i}\right)^{T} Z\left(\sum_{i=1}^{+\infty} a_{i} x_{i}\right)
$$




$$
\leq\left(\sum_{i=1}^{+\infty} a_{i} b_{i}^{-1}\right)\left(\sum_{i=1}^{+\infty} a_{i} b_{i} x_{i}^{T} Z x_{i}\right)
$$

The main objective of this paper is to design the switching controller (8) such that, under the ADT switching signals, the closed-loop error dynamics (9) has the following properties:

1) all trajectories are bounded for admissible initial conditions $\bar{\phi}(\theta)(\theta \in(-\infty, 0])$ and non-zero disturbances $\bar{\omega}(k)$;

2) the $l_{2}-l_{\infty}$ performance requirement

$$
\|e(k)\|_{\infty}^{2} \leq \gamma\|\bar{\omega}(k)\|_{2}^{2}+\gamma V(0)
$$

is ensured, where $\gamma>0$ is a scalar and $V(k)$ is a functional;

3 ) when $\bar{\omega}(k)=0$, the exponential stability can be guaranteed for admissible initial conditions $\bar{\phi}(\theta)(\theta \in(-\infty, 0])$.

Remark 3: Recently, the synchronization control problem has been addressed for dynamical networks subject to actuator saturations [3], [7], [46], [48]. Different from most existing results, this paper attempts to discuss the synchronization control problem for dynamical networks with time delays under actuator saturations in a discrete-time framework. Moreover, the switching topology, the distributed delay and the external disturbances are incorporated in the considered network. On the other hand, it is worth pointing out that, different from the weighted $l_{2}-l_{\infty}$ performance index introduced in [8], [10] for switched dynamical systems, the performance proposed in this paper is in a more appropriate non-weighted framework.

In the sequel, let us introduce the delay-dependent sector conditions for the purpose of dealing with the dead-zone nonlinearity $\psi(u)$ induced by actuator saturations. First, we define the functionals

$$
\begin{aligned}
v_{i}(k)= & U_{i}^{\sigma_{k}} e(k)+V_{i}^{\sigma_{k}} e\left(k-\tau_{k}\right) \\
& +W_{i}^{\sigma_{k}} \sum_{l=1}^{+\infty} \nu_{l} \bar{h}(e(k-l)), i=1,2, \ldots, N
\end{aligned}
$$

where $\sigma_{k}$ is the switching signal as in (1) and (8), and $U_{i}^{\alpha}$, $V_{i}^{\alpha}$ and $W_{i}^{\alpha}(\alpha \in \mathcal{M})$ are $m \times n N$ real constant matrices.

Letting $\epsilon_{i} \in \mathbb{R}^{N}(i \in \mathcal{N})$ be a row vector whose $i$-th element is 1 and others are zero, it can be seen that

$$
u_{i}(k)=\epsilon_{i} \otimes K_{i}^{\sigma_{k}} e(k), i=1,2, \ldots, N .
$$

Assume that the following constraint conditions are true:

$$
\begin{gathered}
\left|u_{i(j)}(k)-v_{i(j)}(k)\right|=\mid\left(\epsilon_{i} \otimes K_{i(j)}^{\sigma_{k}}-U_{i(j)}^{\sigma_{k}}\right) e(k) \\
-V_{i(j)}^{\sigma_{k}} e\left(k-\tau_{k}\right)-W_{i(j)}^{\sigma_{k}} \sum_{l=1}^{+\infty} \nu_{l} \bar{h}(e(k-l)) \mid \leq \bar{u}_{i(l)}, \\
i=1,2, \ldots, N, j=1,2, \ldots, m, k \geq 0 .
\end{gathered}
$$

Then, by using the generalized sector condition in [37], it is concluded that the following sector conditions hold:

$$
\begin{aligned}
& \psi^{T}\left(u_{i}(k)\right) T_{i}^{\sigma_{k}}\left[\psi\left(u_{i}(k)\right)-U_{i}^{\sigma(k)} e(k)\right. \\
& \left.-V_{i}^{\sigma_{k}} e\left(k-\tau_{k}\right)-W_{i}^{\sigma_{k}} \sum_{l=1}^{+\infty} \nu_{l} \bar{h}(e(k-l))\right] \leq 0, \\
& i=1,2, \ldots, N, \quad k \geq 0
\end{aligned}
$$

where $T_{i}^{\alpha}>0(\alpha \in \mathcal{M})$ is any $m \times m$ diagonal matrix.
Denoting that

$$
\begin{aligned}
& T_{\sigma_{k}} \triangleq \operatorname{diag}\left\{T_{1}^{\sigma_{k}}, T_{2}^{\sigma_{k}}, \ldots, T_{N}^{\sigma_{k}}\right\} \\
& U_{\sigma_{k}} \triangleq\left[\begin{array}{llll}
\left(U_{1}^{\sigma_{k}}\right)^{T} & \left(U_{2}^{\sigma_{k}}\right)^{T} & \ldots & \left(U_{N}^{\sigma_{k}}\right)^{T}
\end{array}\right]^{T}, \\
& V_{\sigma_{k}} \triangleq\left[\begin{array}{llll}
\left(V_{1}^{\sigma_{k}}\right)^{T} & \left(V_{2}^{\sigma_{k}}\right)^{T} & \ldots & \left(V_{N}^{\sigma_{k}}\right)^{T}
\end{array}\right]^{T}, \\
& W_{\sigma_{k}} \triangleq\left[\begin{array}{llll}
\left(W_{1}^{\sigma_{k}}\right)^{T} & \left(W_{2}^{\sigma_{k}}\right)^{T} & \ldots & \left(W_{N}^{\sigma_{k}}\right)^{T}
\end{array}\right]^{T},
\end{aligned}
$$

the inequalities in (13) can be represented as

$$
\begin{gathered}
\psi^{T}(u(k)) T_{\sigma_{k}}\left[\psi(u(k))-U_{\sigma_{k}} e(k)-V_{\sigma_{k}} e\left(k-\tau_{k}\right)\right. \\
\left.-W_{\sigma_{k}} \sum_{l=1}^{+\infty} \nu_{l} \bar{h}(e(k-l))\right] \leq 0, k \geq 0 .
\end{gathered}
$$

Remark 4: Under the constraint conditions (12), the novel mixed-delay-dependent sector conditions (13) are proposed in this paper to alleviate the effect of actuator saturations in a less conservative framework. Different from the existing techniques [4], [6], [7], the infinite-distributed-delay terms are specifically introduced, for the first time, in our proposed sector conditions to deal with the infinite distributed delay effectively. Moreover, different from the delay-dependent approach proposed in [6], we introduce only the discrete-delay terms in the sector conditions (13) to tackle the discrete delay in a relatively simple framework, which should be more appropriate for large-scale networks. Using the mixed-delaydependent sector conditions (13), some slack variables can be effectively introduced in the main results of this paper, and this allows us to obtain less conservative results.

\section{MAin RESUlts}

In this section, we will first establish the corresponding analysis condition by employing the ADT approach and the following piecewise Lyapunov-like functional:

$$
\begin{aligned}
V_{\sigma_{k}}(k)= & e^{T}(k) P_{\sigma_{k}} e(k)+\sum_{j=-\bar{\tau}}^{-\underline{\tau}} \sum_{l=k+j}^{k-1} \rho^{k-l-1} e^{T}(l) Q_{\sigma_{k}} e(l) \\
& +\sum_{j=1}^{+\infty} \nu_{j} \sum_{l=k-j}^{k-1} \rho^{k-l-1} \bar{h}^{T}(e(l)) Z_{\sigma_{k}} \bar{h}(e(l)) . \quad(15)
\end{aligned}
$$

where $0<\rho<1, P_{\alpha}>0, Q_{\alpha}>0$ and $Z_{\alpha}>0(\alpha \in \mathcal{M})$.

For convenience of subsequent presentation, we denote

$$
\begin{gathered}
\bar{\nu} \triangleq \sum_{l=1}^{+\infty} \nu_{l}, \hat{\nu} \triangleq \sum_{l=1}^{+\infty} \nu_{l} \rho^{-l}, \hat{\tau} \triangleq \bar{\tau}-\underline{\tau}+1, \\
\kappa_{1} \triangleq \frac{\hat{\tau}(1-\rho)-\rho \tau+\rho^{\bar{\tau}+1}}{(1-\rho)^{2}}, \kappa_{2} \triangleq \sum_{l=1}^{+\infty} \frac{1-\rho^{l}}{1-\rho} \nu_{l}, \\
\hat{H} \triangleq \bar{H}_{1}^{T} \bar{H}_{2}+\bar{H}_{2}^{T} \bar{H}_{1}, \check{H} \triangleq \bar{H}_{1}^{T} \bar{H}_{1}+\bar{H}_{2}^{T} \bar{H}_{2} .
\end{gathered}
$$

Theorem 1: Let the scalars $0<\rho<1, \mu \geq 1, \delta>0$ and the integer $\mathbb{N}_{0} \geq 0$ be given. Assume that there exist $n N \times n N$ matrices $P_{\alpha}>0, Q_{\alpha}>0, Z_{\alpha}>0, m N \times n N$ matrices $K_{\alpha} \triangleq \operatorname{diag}\left\{K_{1}^{\alpha}, K_{2}^{\alpha}, \ldots, K_{N}^{\alpha}\right\}, U_{\alpha} \triangleq\left[\left(U_{1}^{\alpha}\right)^{T} \quad\left(U_{2}^{\alpha}\right)^{T}\right.$ $\left.\cdots\left(U_{N}^{\alpha}\right)^{T}\right]^{T}, V_{\alpha} \triangleq\left[\left(V_{1}^{\alpha}\right)^{T}\left(V_{2}^{\alpha}\right)^{T} \ldots\left(V_{N}^{\alpha}\right)^{T}\right]^{T}, W_{\alpha} \triangleq$ $\left[\left(W_{1}^{\alpha}\right)^{T}\left(W_{2}^{\alpha}\right)^{T} \cdots\left(W_{N}^{\alpha}\right)^{T}\right]^{T}, m N \times m N$ diagonal matrices $T_{\alpha} \triangleq \operatorname{diag}\left\{T_{1}^{\alpha}, T_{2}^{\alpha}, \ldots, T_{N}^{\alpha}\right\}>0$, and the scalars $\varepsilon_{1}^{\alpha}>0$, 
$\varepsilon_{2}^{\alpha}>0, \varepsilon_{3}^{\alpha}>0(\alpha=1,2, \ldots, M), \gamma>0,0<\varpi \leq 1 /\left(\mu^{\mathbb{N}_{0}} \delta\right)$ such that the following matrix inequalities hold:

$$
\begin{aligned}
& {\left[\begin{array}{cc}
\Omega_{\alpha} & \Pi_{\alpha}^{T} \\
\Pi_{\alpha} & -P_{\alpha}^{-1}
\end{array}\right]<0, \forall \alpha \in \mathcal{M},} \\
& P_{\alpha} \leq \mu P_{\beta}, Q_{\alpha} \leq \mu Q_{\beta}, Z_{\alpha} \leq \mu Z_{\beta}, \\
& \forall \alpha, \beta \in \mathcal{M}, \alpha \neq \beta, \\
& {\left[\begin{array}{cccc}
\bar{u}_{i(j)}^{2} \varpi & \epsilon_{i} \otimes K_{i(j)}^{\alpha}-U_{i(j)}^{\alpha} & -V_{i(j)}^{\alpha} & -W_{i(j)}^{\alpha} \\
* & P_{\alpha} & 0 & 0 \\
* & * & \rho^{\bar{\tau}-1} Q_{\alpha} & 0 \\
* & * & * & \frac{1}{\rho \hat{\nu}} Z_{\alpha}
\end{array}\right]>0} \\
& i=1,2, \ldots, N, j=1,2, \ldots, m, \forall \alpha \in \mathcal{M} \\
& {\left[\begin{array}{cc}
\gamma I & I \\
I & P_{\alpha}
\end{array}\right]>0, \forall \alpha \in \mathcal{M}}
\end{aligned}
$$

where

$\Pi_{\alpha}=\left[\begin{array}{llllllll}\bar{C}+\bar{F}_{1}+\bar{L}_{\alpha}+\bar{B} K_{\alpha} & \bar{G}_{1} & \bar{A} & \bar{D}_{1} & 0 & \bar{D}_{2} & -\bar{B} & \bar{E}\end{array}\right]$,

$\Omega_{\alpha}=\left[\begin{array}{cccccccc}\Omega_{11}^{\alpha} & 0 & \Omega_{13}^{\alpha} & 0 & \Omega_{15}^{\alpha} & 0 & \Omega_{17}^{\alpha} & 0 \\ * & \Omega_{22}^{\alpha} & 0 & \Omega_{24}^{\alpha} & 0 & 0 & \Omega_{27}^{\alpha} & 0 \\ * & * & \Omega_{33}^{\alpha} & 0 & 0 & 0 & 0 & 0 \\ * & * & * & \Omega_{44}^{\alpha} & 0 & 0 & 0 & 0 \\ * & * & * & * & \Omega_{55}^{\alpha} & 0 & 0 & 0 \\ * & * & * & * & * & -\frac{1}{\hat{\nu}} Z_{\alpha} & \Omega_{67}^{\alpha} & 0 \\ * & * & * & * & * & * & -2 T_{\alpha} & 0 \\ * & * & * & * & * & * & * & -I\end{array}\right]$

with

$$
\begin{aligned}
& \Omega_{11}^{\alpha}=-\rho P_{\alpha}+\hat{\tau} Q_{\alpha}-\varepsilon_{3}^{\alpha} \hat{H}, \Omega_{13}^{\alpha}=\varepsilon_{1}^{\alpha}\left(\bar{F}_{2}-\bar{F}_{1}\right)^{T}, \\
& \Omega_{15}^{\alpha}=\varepsilon_{3}^{\alpha}\left(\bar{H}_{1}+\bar{H}_{2}\right)^{T}, \Omega_{17}^{\alpha}=U_{\alpha}^{T} T_{\alpha}, \Omega_{22}^{\alpha}=-\rho^{\bar{\tau}} Q_{\alpha}, \\
& \Omega_{24}^{\alpha}=\varepsilon_{2}^{\alpha}\left(\bar{G}_{2}-\bar{G}_{1}\right)^{T}, \Omega_{27}^{\alpha}=V_{\alpha}^{T} T_{\alpha}, \Omega_{33}^{\alpha}=-2 \varepsilon_{1}^{\alpha} I, \\
& \Omega_{44}^{\alpha}=-2 \varepsilon_{2}^{\alpha} I, \Omega_{55}^{\alpha}=-2 \varepsilon_{3}^{\alpha} I+\bar{\nu} Z_{\alpha}, \Omega_{67}^{\alpha}=W_{\alpha}^{T} T_{\alpha} .
\end{aligned}
$$

Then, under the ADT switching with $\mathbb{T}_{a} \geq \mathbb{T}_{a}^{*} \triangleq-\ln \mu / \ln \rho$, the closed-loop error dynamics (9) has the following features: 1) all states remain bounded for all initial conditions $\bar{\phi}(\theta)$ satisfying $V_{\sigma_{0}}(0) \leq 1 /\left(\mu^{\mathbb{N}_{0}} \varpi\right)-\delta$ and all non-zero disturbances $\bar{\omega}(k)$ satisfying $(7) ; 2)$ the $l_{2}-l_{\infty}$ performance requirement $\|e(k)\|_{\infty}^{2} \leq \gamma^{*}\|\bar{\omega}(k)\|_{2}^{2}+\gamma^{*} V_{\sigma_{0}}(0)$ is ensured where $\gamma^{*}=$ $\mu^{\mathbb{N}_{0}} \gamma$; 3) when $\bar{\omega}(k)=0$, all states converge exponentially to the origin for all $\bar{\phi}(\theta)$ satisfying $V_{\sigma_{0}}(0) \leq 1 /\left(\mu^{\mathbb{N}} \varpi\right)$.

Proof: For $\forall \alpha \in \mathcal{M}$, by calculations, one obtains

$$
\begin{aligned}
\Delta V_{\alpha}(k) \triangleq & V_{\alpha}(k+1)-\rho V_{\alpha}(k) \\
\leq & e^{T}(k+1) P_{\alpha} e(k+1)-\rho e^{T}(k) P_{\alpha} e(k) \\
& +\hat{\tau} e^{T}(k) Q_{\alpha} e(k)-\rho^{\bar{\tau}} e^{T}\left(k-\tau_{k}\right) Q_{\alpha} \\
& \times e\left(k-\tau_{k}\right)+\bar{\nu} \bar{h}^{T}(e(k)) Z_{\alpha} \bar{h}(e(k)) \\
& -\sum_{l=1}^{+\infty} \nu_{l} \rho^{l} \bar{h}^{T}(e(k-l)) Z_{\alpha} \bar{h}(e(k-l)) .
\end{aligned}
$$

Using Lemma 1, it can be seen that

$$
\begin{aligned}
& -\sum_{l=1}^{+\infty} \nu_{l} \rho^{l} \bar{h}^{T}(e(k-l)) Z_{\alpha} \bar{h}(e(k-l)) \\
\leq & -\frac{1}{\hat{\nu}}\left[\sum_{l=1}^{+\infty} \nu_{l} \bar{h}(e(k-l))\right]^{T} Z_{\alpha}\left[\sum_{l=1}^{+\infty} \nu_{l} \bar{h}(e(k-l))\right] .
\end{aligned}
$$

For any scalars $\varepsilon_{i}^{\alpha}>0, i=1,2,3$, it follows from (6) that

$$
\begin{aligned}
& -2 \varepsilon_{1}^{\alpha} \bar{f}^{T}(e(k))\left[\bar{f}(e(k))-\left(\bar{F}_{2}-\bar{F}_{1}\right) e(k)\right] \geq 0, \\
& -2 \varepsilon_{2}^{\alpha} \bar{g}^{T}\left(e\left(k-\tau_{k}\right)\right) \\
& \quad \times\left[\bar{g}\left(e\left(k-\tau_{k}\right)\right)-\left(\bar{G}_{2}-\bar{G}_{1}\right) e\left(k-\tau_{k}\right)\right] \geq 0, \\
& -2 \varepsilon_{3}^{\alpha}\left[\bar{h}(e(k))-\bar{H}_{1} e(k)\right]^{T}\left[\bar{h}(e(k))-\bar{H}_{2} e(k)\right] \geq 0 .
\end{aligned}
$$

Under the constraints (12), it is seen from (14) that

$$
\begin{aligned}
& -2 \psi^{T}(u(k)) T_{\alpha}\left[\psi(u(k))-U_{\alpha} e(k)\right. \\
& \left.\quad-V_{\alpha} e\left(k-\tau_{k}\right)-W_{\alpha} \sum_{l=1}^{+\infty} \mu_{l} \bar{h}(e(k-l))\right] \geq 0 .
\end{aligned}
$$

Adding the left-hand sides of the inequalities (22)-(25) to $\Delta V_{\alpha}(k)$ and using the inequality (21), one eventually obtains

$$
\Delta V_{\alpha}(k) \leq \xi^{T}(k)\left(\Omega_{\alpha}+\Pi_{\alpha}^{T} P_{\alpha} \Pi_{\alpha}\right) \xi(k)+\bar{\omega}^{T}(k) \bar{\omega}(k),
$$

where $\Omega_{\alpha}, \Pi_{\alpha}$ have the same definitions as in (16) and

$$
\begin{aligned}
& \xi(k)=\left[\begin{array}{llll}
e^{T}(k) & e^{T}\left(k-\tau_{k}\right) & \bar{f}^{T}(e(k)) & \bar{g}^{T}\left(e\left(k-\tau_{k}\right)\right)
\end{array}\right. \\
& \left.\bar{h}^{T}(e(k)) \sum_{l=1}^{+\infty} \nu_{l} \bar{h}^{T}(e(k-l)) \quad \psi^{T}(u(k)) \bar{\omega}^{T}(k)\right]^{T} .
\end{aligned}
$$

Applying Schur complement to (16), it is clear that $\Omega_{\alpha}+$ $\Pi_{\alpha}^{T} P_{\alpha} \Pi_{\alpha}<0, \forall \alpha \in \mathcal{M}$. Then, it follows from (26) that

$$
V_{\alpha}(k+1) \leq \rho V_{\alpha}(k)+\bar{\omega}^{T}(k) \bar{\omega}(k), \forall \alpha \in \mathcal{M} .
$$

Also, it is seen from (17) that

$$
V_{\alpha}(k) \leq \mu V_{\beta}(k), \forall \alpha, \beta \in \mathcal{M} .
$$

Let $0=k_{0}<k_{1}<\cdots<k_{s}<\cdots$ be any switching time sequence and $k$ be any positive integer belonging to $\left[k_{s}, k_{s+1}\right)$. If $k-k_{s} \geq 1$, using the inequalities (27) and (28) recursively, we can deduce the following inequality:

$$
\begin{aligned}
& V_{\sigma_{k}}(k) \leq \mu^{s} \rho^{k} V_{\sigma_{0}}(0)+\sum_{l=k_{s}}^{k-1} \rho_{k l} \bar{\omega}^{T}(l) \bar{\omega}(l) \\
& \quad+\mu \sum_{l=k_{s-1}}^{k_{s}-1} \rho_{k l} \bar{\omega}^{T}(l) \bar{\omega}(l)+\cdots+\mu^{s} \sum_{l=k_{0}}^{k_{1}-1} \rho_{k l} \bar{\omega}^{T}(l) \bar{\omega}(l) \\
& \triangleq \mu^{\mathbb{N}_{\sigma}(0, k)} \rho^{k} V_{\sigma_{0}}(0)+\sum_{l=k_{0}}^{k-1} \mu^{\mathbb{N}_{\sigma}(l, k-1)} \rho_{k l} \bar{\omega}^{T}(l) \bar{\omega}(l)
\end{aligned}
$$

where $\rho_{k l} \triangleq \rho^{k-l-1}$. Similarly, if $k=k_{s}$, one can obtain

$$
\begin{aligned}
& V_{\sigma_{k}}(k) \leq \mu^{s} \rho^{k} V_{\sigma_{0}}(0)+\sum_{l=k_{s-1}}^{k-1} \rho_{k l} \bar{\omega}^{T}(l) \bar{\omega}(l) \\
& \quad+\mu \sum_{l=k_{s-2}}^{k_{s-1}-1} \rho_{k l} \bar{\omega}^{T}(l) \bar{\omega}(l)+\cdots+\mu^{s-1} \sum_{l=k_{0}}^{k_{1}-1} \rho_{k l} \bar{\omega}^{T}(l) \bar{\omega}(l) \\
& \triangleq \mu^{\mathbb{N}_{\sigma}(0, k)} \rho^{k} V_{\sigma_{0}}(0)+\sum_{l=k_{0}}^{k-1} \mu^{\mathbb{N}_{\sigma}(l, k-1)} \rho_{k l} \bar{\omega}^{T}(l) \bar{\omega}(l) .
\end{aligned}
$$

Noting the relationships $\mathbb{N}_{\sigma}(0, k) \leq \mathbb{N}_{0}+k / \mathbb{T}_{a}, \mathbb{N}_{\sigma}(l, k-$ 1) $\leq \mathbb{N}_{0}+(k-l-1) / \mathbb{T}_{a}$ and $\mathbb{T}_{a} \geq \mathbb{T}_{a}^{*} \triangleq-\ln \mu / \ln \rho$, we have

$$
\mu^{\mathbb{N}_{\sigma}(0, k)} \rho^{k} \leq \mu^{\mathbb{N}_{0}}\left(\mu^{1 / \mathbb{T}_{a}} \rho\right)^{k} \triangleq \mu^{\mathbb{N}_{0}} \vartheta^{k} \leq \mu^{\mathbb{N}_{0}},
$$




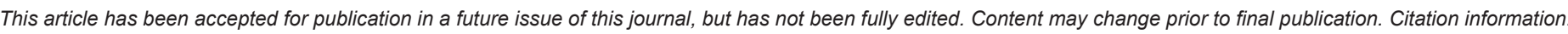
DOI10.1109/TNNLS.2020.2996094, IEEE Transactions on Neural Networks and Learning Systems

$$
\mu^{\mathbb{N}_{\sigma}(l, k-1)} \rho^{k-l-1} \leq \mu^{\mathbb{N}_{0}}\left(\mu^{1 / \mathbb{T}_{a}} \rho\right)^{k-l-1} \leq \mu^{\mathbb{N}_{0}} .
$$

Then, for $\forall k \in\left[k_{s}, k_{s+1}\right)$, it follows from (29) and (30) that

$$
V_{\sigma_{k}}(k) \leq \mu^{\mathbb{N}_{0}} \vartheta^{k} V_{\sigma_{0}}(0)+\mu^{\mathbb{N}_{0}} \sum_{k=0}^{+\infty} \bar{\omega}^{T}(k) \bar{\omega}(k) .
$$

On the other hand, for $\forall \alpha \in \mathcal{M}$, it is seen from (15) that

$$
\begin{aligned}
V_{\alpha}(k) \geq & e^{T}(k) P_{\alpha} e(k)+\sum_{l=k-\bar{\tau}}^{k-\tau} \rho^{k-l-1} e^{T}(l) Q_{\alpha} e(l) \\
& +\sum_{l=1}^{+\infty} \nu_{l} \rho^{l-1} \bar{h}^{T}(e(k-l)) Z_{\alpha} \bar{h}(e(k-l)) \\
\geq & e^{T}(k) P_{\alpha} e(k)+\rho^{\bar{\tau}-1} e^{T}\left(k-\tau_{k}\right) Q_{\alpha} e\left(k-\tau_{k}\right) \\
& +\frac{1}{\rho \hat{\nu}}\left[\sum_{l=1}^{+\infty} \nu_{l} \bar{h}(e(k-l))\right]^{T} Z_{\alpha}\left[\sum_{l=1}^{+\infty} \nu_{l} \bar{h}(e(k-l))\right] .
\end{aligned}
$$

Applying Schur complement to (18), one obtains

$$
\begin{aligned}
& {\left[\begin{array}{ccc}
P_{\alpha} & 0 & 0 \\
* & \rho^{\bar{\tau}-1} Q_{\alpha} & 0 \\
* & * & \frac{1}{\rho \hat{\nu}} Z_{\alpha}
\end{array}\right]>\frac{1}{\bar{u}_{i(j)}^{2} \varpi}\left(\Phi_{i(j)}^{\alpha}\right)^{T} \Phi_{i(j)}^{\alpha},} \\
& i=1,2, \ldots, N, \quad j=1,2, \ldots, m, \forall \alpha \in \mathcal{M},
\end{aligned}
$$

where $\Phi_{i(j)}^{\alpha}=\left[\epsilon_{i} \otimes K_{i(j)}^{\alpha}-U_{i(j)}^{\alpha} \quad-V_{i(j)}^{\alpha} \quad-W_{i(j)}^{\alpha}\right]$. Then, from (34) and (35), it follows that

$$
\begin{aligned}
V_{\sigma_{k}}(k) & \geq \frac{1}{\bar{u}_{i(j)}^{2} \varpi} \eta^{T}(k)\left(\Phi_{i(j)}^{\sigma(k)}\right)^{T} \Phi_{i(j)}^{\sigma(k)} \eta(k) \\
& =\frac{1}{\bar{u}_{i(j)}^{2} \varpi}\left|u_{i(j)}(k)-v_{i(j)}(k)\right|^{2}, \\
i & =1,2, \ldots, N, j=1,2, \ldots, m
\end{aligned}
$$

where $\eta(k)=\left[\begin{array}{llll}e^{T}(k) & e^{T}\left(k-\tau_{\underline{k}}\right) & \sum_{l=1}^{+\infty} \nu_{l} \bar{h}^{T}(e(k-l))\end{array}\right]^{T}$.

For all initial conditions $\bar{\phi}(\theta)$ satisfying $V_{\sigma_{0}}(0) \leq$ $1 /\left(\mu^{\mathbb{N}_{0}} \varpi\right)-\delta$ and all non-zero $\bar{\omega}(k)$ satisfying (7), it is seen from (7), (33) and (36) that the constraints in (12) can be ensured. Furthermore, one can obtain from (15) and (33) that

$$
\min _{\alpha \in \mathcal{M}} \lambda_{m}\left(P_{\alpha}\right)\|e(k)\|_{2}^{2} \leq V_{\sigma_{k}}(k) \leq 1 / \varpi, k \geq 0
$$

which implies that all error states of (9) are bounded.

Next, we will show that the $l_{2}-l_{\infty}$ performance requirement of the closed-loop dynamics (9) can be guaranteed. Using Schur complement, the inequalities in (19) hold if and only if

$$
P_{\alpha}>\frac{1}{\gamma} I, \alpha \in \mathcal{M}
$$

From (15), (33) and (38), it is obvious that

$$
e^{T}(k) e(k) \leq \gamma^{*}\|\bar{\omega}(k)\|_{2}^{2}+\gamma^{*} V_{\sigma_{0}}(0), k \geq 0 .
$$

Taking the supremum on both sides of (39), it is seen that the $l_{2}-l_{\infty}$ performance constraint is ensured for all $\bar{\phi}(\theta)$ satisfying $V_{\sigma_{0}}(0) \leq 1 /\left(\mu^{\mathbb{N}_{0}} \varpi\right)-\delta$ and all non-zero $\bar{\omega}(k)$ satisfying (7).

Finally, it remains to prove that, when $\bar{\omega}(k)=0$, all states of the error dynamics (9) converge exponentially to the origin for all $\bar{\phi}(\theta)$ satisfying $V_{\sigma_{0}}(0) \leq 1 /\left(\mu^{\mathbb{N}_{0}} \varpi\right)$. When $\bar{\omega}(k)=0$, similar to the above argument, it is clear that the constraints in (12) can also be guaranteed for all $\bar{\phi}(\theta)$ satisfying $V_{\sigma_{0}}(0) \leq 1 /\left(\mu^{\mathbb{N}_{0}} \varpi\right)$. Moreover, when $\bar{\omega}(k)=0$, the inequality (33) becomes $V_{\sigma_{k}}(k) \leq \mu^{\mathbb{N}_{0}} \vartheta^{k} V_{\sigma(0)}(0)$. Noting that $\vartheta=\mu^{1 / \mathbb{T}_{a}} \rho \leq 1$ under the condition $\mathbb{T}_{a} \geq \mathbb{T}_{a}^{*} \triangleq-\ln \mu / \ln \rho$, it can be concluded that the closed-loop error dynamics (9) is locally exponentially stable and this completes the proof.

Remark 5: It is seen from the proof of Theorem 1 that, different from continuous-time case [4], [7], the discretetime characteristics are extensively explored in this paper and the important relationship (34) is subtly established. In this case, the mixed-delay-dependent sector conditions (13) can be effectively utilized even if we choose the rather simple Lyapunov-like functional (15). As a result, the slack variables $V_{\alpha}$ and $W_{\alpha}(\alpha=1,2, \ldots, M)$ are additionally introduced in the conditions of Theorem 1 without bringing extra constraints.

Based on the Theorem 1, in the subsequent part, we will consider the control design problem within an LMI framework.

Theorem 2: Let the scalars $0<\rho<1, \mu \geq 1$, $\delta>0, \varphi_{\alpha}, \psi_{\alpha}$ and the integer $\mathbb{N}_{0} \geq 0$ be given. Assume that there exist $n N \times n N$ matrices $\bar{P}_{\alpha}>0, \bar{Q}_{\alpha}>0$, $\bar{Z}_{\alpha}>0, X \triangleq \operatorname{diag}\left\{X_{1}, X_{2}, \ldots, X_{N}\right\}, m N \times n N$ matrices $Y_{\alpha} \triangleq \operatorname{diag}\left\{Y_{1}^{\alpha}, Y_{2}^{\alpha}, \ldots, Y_{N}^{\alpha},\right\}, \bar{U}_{\alpha} \triangleq\left[\left(\bar{U}_{1}^{\alpha}\right)^{T} \quad\left(\bar{U}_{2}^{\alpha}\right)^{T} \ldots\right.$ $\left.\left(\bar{U}_{N}^{\alpha}\right)^{T}\right]^{T}, \bar{V}_{\alpha} \triangleq\left[\left(\bar{V}_{1}^{\alpha}\right)^{T}\left(\bar{V}_{2}^{\alpha}\right)^{T} \cdots\left(\bar{V}_{N}^{\alpha}\right)^{T}\right]^{T}, \bar{W}_{\alpha} \triangleq$ $\left[\left(\bar{W}_{1}^{\alpha}\right)^{T}\left(\bar{W}_{2}^{\alpha}\right)^{T} \cdots\left(\bar{W}_{N}^{\alpha}\right)^{T}\right]^{T}, m N \times m N$ diagonal matrices $\bar{T}_{\alpha} \triangleq \operatorname{diag}\left\{\bar{T}_{1}^{\alpha}, \bar{T}_{2}^{\alpha}, \ldots, \bar{T}_{N}^{\alpha}\right\}>0$, and the scalars $\bar{\varepsilon}_{1}^{\alpha}>0$, $\bar{\varepsilon}_{2}^{\alpha}>0, \bar{\varepsilon}_{3}^{\alpha}>0(\alpha=1,2, \ldots, M), \gamma>0,0<\varpi \leq 1 /\left(\mu^{\mathbb{N} 0} \delta\right)$ such that the following LMIs hold:

$$
\begin{aligned}
& {\left[\begin{array}{ccc}
\Sigma_{\alpha} & \Upsilon_{\alpha}^{T} & \Psi_{\alpha}^{T} \\
* & \Theta_{\alpha} & 0 \\
* & * & -\frac{1}{\bar{\nu}} \bar{Z}_{\alpha}
\end{array}\right]<0, \forall \alpha \in \mathcal{M},} \\
& \bar{P}_{\alpha} \leq \mu \bar{P}_{\beta}, \bar{Q}_{\alpha} \leq \mu \bar{Q}_{\beta}, \bar{Z}_{\alpha} \leq \mu \bar{Z}_{\beta}, \\
& \forall \alpha, \beta \in \mathcal{M}, \alpha \neq \beta, \\
& {\left[\begin{array}{cccc}
\bar{u}_{i(j)}^{2} \varpi & \epsilon_{i} \otimes Y_{i(j)}^{\alpha}-\bar{U}_{i(j)}^{\alpha} & -\bar{V}_{i(j)}^{\alpha} & -\bar{W}_{i(j)}^{\alpha} \\
* & \bar{P}_{\alpha} & 0 & 0 \\
* & * & \rho^{\bar{\tau}-1} \bar{Q}_{\alpha} & 0 \\
* & * & * & \frac{1}{\rho \hat{\nu}} \bar{Z}_{\alpha}
\end{array}\right]>0} \\
& i=1,2, \ldots, N, j=1,2, \ldots, m, \forall \alpha \in \mathcal{M} \\
& {\left[\begin{array}{cc}
\gamma I & X^{T} \\
X & \bar{P}_{\alpha}
\end{array}\right]>0, \forall \alpha \in \mathcal{M}}
\end{aligned}
$$

where

$$
\Sigma_{\alpha}=\left[\begin{array}{ccccccccc}
\Sigma_{11}^{\alpha} & 0 & \Sigma_{13}^{\alpha} & 0 & \Sigma_{15}^{\alpha} & 0 & \bar{U}_{\alpha}^{T} & 0 & \Sigma_{19}^{\alpha} \\
* & \Sigma_{22}^{\alpha} & 0 & \Sigma_{24}^{\alpha} & 0 & 0 & \bar{V}_{\alpha}^{T} & 0 & \Sigma_{29}^{\alpha} \\
* & * & \Sigma_{33}^{\alpha} & 0 & 0 & 0 & 0 & 0 & \Sigma_{39}^{\alpha} \\
* & * & * & \Sigma_{44}^{\alpha} & 0 & 0 & 0 & 0 & \Sigma_{49}^{\alpha} \\
* & * & * & * & \Sigma_{55}^{\alpha} & 0 & 0 & 0 & 0 \\
* & * & * & * & * & \Sigma_{66}^{\alpha} & \bar{W}_{\alpha}^{T} & 0 & \Sigma_{69}^{\alpha} \\
* & * & * & * & * & * & \Sigma_{77}^{\alpha} & 0 & \Sigma_{79}^{\alpha} \\
* & * & * & * & * & * & * & -I & \bar{E}^{T} \\
* & * & * & * & * & * & * & * & \Sigma_{99}^{\alpha}
\end{array}\right]
$$

and

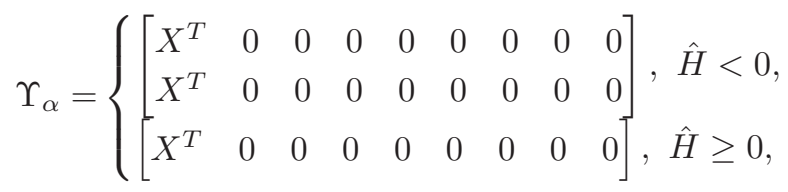




$$
\begin{aligned}
& \Theta_{\alpha}=\left\{\begin{array}{l}
{\left[\begin{array}{cc}
-\bar{Q}_{\alpha} / \hat{\tau} & 0 \\
0 & \bar{\varepsilon}_{3}^{\alpha} \hat{H}^{-1}
\end{array}\right], \hat{H}<0,} \\
-\bar{Q}_{\alpha} / \hat{\tau}, \hat{H} \geq 0,
\end{array}\right. \\
& \Psi_{\alpha}=\left[\begin{array}{lllllllll}
0 & 0 & 0 & 0 & \bar{\varepsilon}_{3}^{\alpha} I & 0 & 0 & 0 & 0
\end{array}\right]
\end{aligned}
$$

with

$$
\begin{aligned}
& \Sigma_{11}^{\alpha}=\left\{\begin{array}{l}
-\rho \bar{P}_{\alpha}, \hat{H} \leq 0, \\
-\rho \bar{P}_{\alpha}-\varphi_{\alpha}\left(X+X^{T}\right)+\varphi_{\alpha}^{2} \bar{\varepsilon}_{3}^{\alpha} \hat{H}^{-1}, \hat{H}>0,
\end{array}\right. \\
& \Sigma_{13}^{\alpha}=X\left(\bar{F}_{2}-\bar{F}_{1}\right)^{T}, \Sigma_{15}^{\alpha}=X\left(\bar{H}_{1}+\bar{H}_{2}\right)^{T}, \\
& \Sigma_{19}^{\alpha}=X\left(\bar{C}+\bar{F}_{1}+\bar{L}_{\alpha}\right)^{T}+Y_{\alpha}^{T} \bar{B}^{T}, \Sigma_{22}^{\alpha}=-\rho^{\bar{\tau}} \bar{Q}_{\alpha}, \\
& \Sigma_{24}^{\alpha}=\bar{Q}_{\alpha}\left(\bar{G}_{2}-\bar{G}_{1}\right)^{T}, \Sigma_{29}^{\alpha}=\bar{Q}_{\alpha} \bar{G}_{1}^{T}, \Sigma_{33}^{\alpha}=-2 \bar{\varepsilon}_{1}^{\alpha} I, \\
& \Sigma_{39}^{\alpha}=\bar{\epsilon}_{1}^{\alpha} \bar{A}^{T}, \Sigma_{44}^{\alpha}=-2 \bar{\varepsilon}_{2}^{\alpha} I, \Sigma_{49}^{\alpha}=\bar{\epsilon}_{2}^{\alpha} \bar{D}_{1}^{T}, \Sigma_{55}^{\alpha}=-2 \bar{\varepsilon}_{3}^{\alpha} I, \\
& \Sigma_{66}^{\alpha}=-\bar{Z}_{\alpha} / \hat{\nu}, \Sigma_{69}^{\alpha}=\bar{Z}_{\alpha} \bar{D}_{2}^{T}, \Sigma_{77}^{\alpha}=-2 \bar{T}_{\alpha}, \\
& \Sigma_{79}^{\alpha}=-\bar{T}_{\alpha} \bar{B}^{T}, \Sigma_{99}^{\alpha}=-\psi_{\alpha}\left(X+X^{T}\right)+\psi_{\alpha}^{2} \bar{P}_{\alpha} .
\end{aligned}
$$

Then, under the ADT switching with $\mathbb{T}_{a} \geq \mathbb{T}_{a}^{*} \triangleq-\ln \mu / \ln \rho$, there exists a switching controller of the form (8) with $K_{i}^{\alpha}=Y_{i}^{\alpha} X_{i}^{-T}$ such that: 1) all error states are bounded for all initial conditions $\bar{\phi}(\theta)$ satisfying $V_{\sigma_{0}}(0) \leq 1 /\left(\mu^{\mathbb{N}_{0}} \varpi\right)-\delta$ and all non-zero $\bar{\omega}(k)$ satisfying (7); 2) $l_{2}-l_{\infty}$ performance constraint $\|e(k)\|_{\infty}^{2} \leq \gamma^{*}\|\bar{\omega}(k)\|_{2}^{2}+\gamma^{*} V_{\sigma_{0}}(0)$ is ensured where $\gamma^{*}=\mu^{\mathbb{N}_{0}} \gamma$; 3) when $\bar{\omega}(k)=0$, the error dynamics (9) is exponentially stable for all $\bar{\phi}(\theta)$ satisfying $V_{\sigma_{0}}(0) \leq 1 /\left(\mu^{\mathbb{N}_{0}} \varpi\right)$.

Proof: First of all, it is seen from LMIs (40) that $\Sigma_{99}^{\alpha}=$ $-\psi_{\alpha}\left(X+X^{T}\right)+\psi_{\alpha}^{2} \bar{P}_{\alpha}<0, \forall \alpha \in \mathcal{M}$. Noting that $\bar{P}_{\alpha}>0$, it can be concluded that the matrix $X$ is invertible if the LMIs in Theorem 2 hold. Then, we can define the following variables:

$$
\left\{\begin{array}{l}
P_{\alpha} \triangleq X^{-1} \bar{P}_{\alpha} X^{-T}, Q_{\alpha} \triangleq \bar{Q}_{\alpha}^{-1}, Z_{\alpha} \triangleq \bar{Z}_{\alpha}^{-1} \\
K_{\alpha} \triangleq Y_{\alpha} X^{-T}, U_{\alpha} \triangleq \bar{U}_{\alpha} X^{-T}, V_{\alpha} \triangleq \bar{V}_{\alpha} \bar{Q}_{\alpha}^{-1} \\
W_{\alpha} \triangleq \bar{W}_{\alpha} \bar{Z}_{\alpha}^{-1}, T_{\alpha} \triangleq \bar{T}_{\alpha}^{-1}, \varepsilon_{1}^{\alpha} \triangleq\left(\bar{\varepsilon}_{1}^{\alpha}\right)^{-1} \\
\varepsilon_{2}^{\alpha} \triangleq\left(\bar{\varepsilon}_{2}^{\alpha}\right)^{-1}, \varepsilon_{3}^{\alpha} \triangleq\left(\bar{\varepsilon}_{3}^{\alpha}\right)^{-1}, \alpha=1,2, \ldots, M
\end{array}\right.
$$

Using Schur complement, it is clear that the LMIs (40) hold if and only if the following matrix inequalities are true:

$$
\Sigma_{\alpha}+\Upsilon_{\alpha}^{T} \Theta_{\alpha}^{-1} \Upsilon_{\alpha}+\bar{\nu} \Upsilon_{\alpha}^{T} \bar{Z}_{\alpha}^{-1} \Upsilon_{\alpha}<0, \forall \alpha \in \mathcal{M}
$$

Pre- and post-multiplying the matrix inequalities (45) by $\operatorname{diag}\left\{X^{-1}, Q_{\alpha}, \varepsilon_{1}^{\alpha} I, \varepsilon_{2}^{\alpha} I, \varepsilon_{3}^{\alpha} I, Z_{\alpha}, T_{\alpha}, I, I\right\}$ and its transpose, and using notations in (44) and the following facts:

$$
\left\{\begin{array}{l}
-\varepsilon_{3}^{\alpha} X \hat{H} X^{T} \leq-\varphi_{\alpha}\left(X+X^{T}\right)+\varphi_{\alpha}^{2} \bar{\varepsilon}_{3}^{\alpha} \hat{H}^{-1}, \\
-X \bar{P}_{\alpha}^{-1} X^{T} \leq-\psi_{\alpha}\left(X+X^{T}\right)+\psi_{\alpha}^{2} \bar{P}_{\alpha}
\end{array}\right.
$$

where $\hat{H}>0$, it is seen that the inequalities (16) are ensured.

On the other hand, performing the corresponding congruence transformations to LMIs (41)-(43), respectively, it is seen that the matrix inequalities (17)-(19) are easily obtained.

Remark 6: Note that the matrix $\hat{H} \triangleq \bar{H}_{1}^{T} \bar{H}_{2}+\bar{H}_{2}^{T} \bar{H}_{1}$ might be positive definite, zero or negative definite. In obtaining LMIs (40) of Theorem 2, three cases of $\hat{H}$ are all considered. Also, in order to reduce the potential conservatism, the adjusting parameters $\varphi_{\alpha}$ and $\psi_{\alpha}(\alpha=1,2, \ldots, M)$ are introduced in Theorem 2. On the other hand, it is worth mentioning that the pinning control strategy can be incorporated in this paper. Without loss of generality, we can select and pin the first $l$
$(1<\check{l}<N)$ nodes of the network. In this case, the matrices $Y_{\alpha}, \bar{U}_{\alpha}, \bar{V}_{\alpha}$ and $\bar{W}_{\alpha}$ in Theorem 2 should be modified as

$$
\begin{aligned}
& Y_{\alpha} \triangleq \operatorname{diag}\left\{Y_{1}^{\alpha}, \ldots, Y_{\grave{l}}^{\alpha}, 0, \ldots, 0\right\},
\end{aligned}
$$

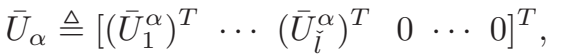

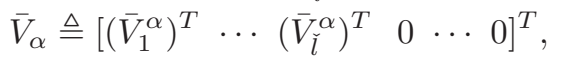

$$
\begin{aligned}
& \bar{W}_{\alpha} \triangleq\left[\begin{array}{llllll}
\left(\bar{W}_{1}^{\alpha}\right)^{T} & \cdots & \left(\bar{W}_{\bar{l}}^{\alpha}\right)^{T} & 0 & \cdots & 0
\end{array}\right]^{T} .
\end{aligned}
$$

Finally, let us tackle the optimization problems involved in Theorem 2. For saturated control systems with external disturbances, a basic problem is to measure the largest disturbance tolerance capability such that all state trajectories are bounded for admissible initial conditions and disturbances. Here, the initial condition of the error dynamics (9) is selected as $\bar{\phi}(\theta)=0(\theta \leq 0)$. In this case, the scalar $\varpi$ in (42) should be replaced by $1 /\left(\mu^{\mathbb{N}_{0}} \delta\right)$. Then, the maximization problem of the disturbance tolerance capability can be described as

$$
\begin{gathered}
\text { Prob.1. } \max _{\bar{P}_{\alpha}, \bar{Q}_{\alpha}, \bar{Z}_{\alpha}, X, Y_{\alpha}, \bar{U}_{\alpha}, \bar{V}_{\alpha}, \bar{W}_{\alpha}, \bar{T}_{\alpha}, \bar{\varepsilon}_{1}^{\alpha}, \bar{\varepsilon}_{2}^{\alpha}, \bar{\varepsilon}_{3}^{\alpha}, \delta} \delta \text {, s.t. }, \\
\text { LMIs (40) - (42) hold. }
\end{gathered}
$$

Once the maximum disturbance tolerance level $\delta_{M}$ is obtained, setting $\delta \leq \delta_{M}$, the minimization of the $l_{2}-l_{\infty}$ performance level $\gamma^{*}=\mu^{\mathbb{N}_{0}} \gamma>0$ can be written as

$$
\begin{gathered}
\text { Prob.2. } \min _{\bar{P}_{\alpha}, \bar{Q}_{\alpha}, \bar{Z}_{\alpha}, X, Y_{\alpha}, \bar{U}_{\alpha}, \bar{V}_{\alpha}, \bar{W}_{\alpha}, \bar{T}_{\alpha}, \bar{\varepsilon}_{1}^{\alpha}, \bar{\varepsilon}_{2}^{\alpha}, \bar{\varepsilon}_{3}^{\alpha}, \gamma} \gamma \text {, s.t., } \\
\text { LMIs (40) - (43) hold. }
\end{gathered}
$$

For the case that $\bar{\omega}(k)=0$, we can maximize the initial condition set when designing the controller (8). In this context, the rows and columns concerning the disturbances in LMIs (40) should be removed. Moreover, without loss of generality, the scalar $\varpi$ in LMIs (42) can be selected as $\varpi=1 / \mu^{\mathbb{N}_{0}}$.

In this paper, we represent the initial condition set as

$$
\mathscr{X}_{r} \triangleq\left\{\bar{\phi}(\theta): \max _{\theta \in(-\infty, 0]}\|\bar{\phi}(\theta)\| \leq r\right\}
$$

where $r$ is a positive scalar to be maximized.

From the third inequality in (6), it is seen that

$$
\begin{aligned}
& 2 \bar{h}^{T}(e(k)) \bar{h}(e(k)) \\
\leq & 2 e^{T}(k)\left(\bar{H}_{1}+\bar{H}_{2}\right)^{T} \bar{h}(e(k))-2 e^{T}(k) \bar{H}_{1}^{T} \bar{H}_{2} e(k) .
\end{aligned}
$$

Note that the following fact:

$$
\begin{aligned}
& 2 e^{T}(k)\left(\bar{H}_{1}+\bar{H}_{2}\right)^{T} \bar{h}(e(k)) \\
\leq & e^{T}(k)\left(\bar{H}_{1}+\bar{H}_{2}\right)^{T}\left(\bar{H}_{1}+\bar{H}_{2}\right) e(k)+\bar{h}^{T}(e(k)) \bar{h}(e(k)) \\
= & e^{T}(k) \check{H} e(k)+2 e^{T}(k) \bar{H}_{1}^{T} \bar{H}_{2} e(k)+\bar{h}^{T}(e(k)) \bar{h}(e(k)) .
\end{aligned}
$$

Then, one can obtain from (48) that

$$
\bar{h}^{T}(e(k)) \bar{h}(e(k)) \leq e^{T}(k) \check{H} e(k) .
$$

Moreover, it follows from (15), (44), (47) and (50) that

$$
\begin{gathered}
V_{\sigma(0)}(0) \leq \max _{\alpha \in \mathcal{M}}\left[\lambda_{M}\left(X^{-1} \bar{P}_{\alpha} X^{-T}\right)+\kappa_{1} \lambda_{M}\left(\bar{Q}_{\alpha}^{-1}\right)\right. \\
\left.+\kappa_{2} \lambda_{M}(\check{H}) \lambda_{M}\left(\bar{Z}_{\alpha}^{-1}\right)\right] r^{2} \triangleq \chi_{r} .
\end{gathered}
$$

Let us introduce the following matrix inequalities:

$$
X^{-1} \bar{P}_{\alpha} X^{-T} \leq p I, \forall \alpha \in \mathcal{M},
$$




$$
\bar{Q}_{\alpha}^{-1} \leq q I, \bar{Z}_{\alpha}^{-1} \leq z I, \forall \alpha \in \mathcal{M}
$$

where $p, q$ and $z$ are some positive scalars. Noting that the inequality (52) is equivalent to $X^{T} \bar{P}_{\alpha}^{-1} X \geq p^{-1} I$ and using the fact $X^{T} \bar{P}_{\alpha}^{-1} X \geq \iota_{\alpha}\left(X+X^{T}\right)-\iota_{\alpha}^{2} \bar{P}_{\alpha}\left(\iota_{\alpha}\right.$ is a scalar $)$, it is concluded that the inequality (52) can be guaranteed by

$$
X+X^{T}-\bar{P}_{\alpha} \geq p^{-1} I, \forall \alpha \in \mathcal{M} \text {. }
$$

Then, applying Schur complement to (53) and (54), it is clear that (52) and (53) are ensured by the following LMIs:

$$
\begin{aligned}
& {\left[\begin{array}{cc}
p I & I \\
I & \iota_{\alpha}\left(X+X^{T}\right)-\iota_{\alpha}^{2} \bar{P}_{\alpha}
\end{array}\right] \geq 0, \forall \alpha \in \mathcal{M},} \\
& {\left[\begin{array}{cc}
q I & I \\
I & \bar{Q}_{\alpha}
\end{array}\right] \geq 0, \quad\left[\begin{array}{cc}
z I & I \\
I & \bar{Z}_{\alpha}
\end{array}\right] \geq 0, \forall \alpha \in \mathcal{M} .}
\end{aligned}
$$

From the above analysis, it is seen that the optimization problem concerning the set $\mathscr{X}_{r}$ can be represented as

$$
\begin{aligned}
& \text { Prob.3. } \min _{\bar{P}_{\alpha}, \bar{Q}_{\alpha}, \bar{Z}_{\alpha}, X, Y_{\alpha}, \bar{U}_{\alpha}, \bar{V}_{\alpha}, \bar{W}_{\alpha}, \bar{T}_{\alpha}, \bar{\varepsilon}_{1}^{\alpha}, \bar{\varepsilon}_{2}^{\alpha}, \bar{\varepsilon}_{3}^{\alpha}, p, q, z} \zeta \text {, s.t., } \\
& \text { LMIs (40) - (42) and (55) - (56) hold }
\end{aligned}
$$

where $\zeta=p+\kappa_{1} q+\kappa_{2} \lambda_{M}(\check{H}) q$. Furthermore, if there exists the optimization solution, the upper bound $r$ of the initial condition set $\mathscr{X}_{r}$ can be obtained by the equation $\chi_{r}=1$.

Remark 7: In the sector conditions (13), by deleting the delay-dependent terms, we can obtain the delay-independent sector conditions. If the same research is conducted by using such delay-independent conditions, the corresponding optimization problems are readily obtained by setting $\bar{V}_{\alpha}=0$ and $\bar{W}_{\alpha}=0(\forall \alpha \in \mathcal{M})$ in LMIs (40) and (42), which are referred to as Prob.1', Prob.2' and Prob.3', respectively, for ease of reference. In addition, it is worth mentioning that, for the case without switching topology and the case with one type of time delay, the corresponding results can also be easily obtained by removing some terms that are no longer relevant.

Remark 8: In this paper, the synchronization control problem is addressed for the first time, for dynamical networks with both switching topology and time delays under actuator saturations within a discrete-time framework. The main results established in Theorems 1 and 2 distinguish themselves from existing literature in the following three aspects: 1) the model considered is comprehensive that involves discretetime dynamics, switching topology, mixed-time-delays and actuator saturations; 2) the proposed mixed-delay-dependent sector conditions are new, which are particularly suitable for analyzing large-scale networks; and 3 ) the proposed $l_{2}$ $l_{\infty}$ performance index is new, which is evaluated in a nonweighted framework. Overall, our work represents the first attempt to deal with the discrete-time dynamical networks with mixed delays and switching topology where the actuator saturations are specifically considered in designing controllers.

Remark 9: In this paper, the techniques handling the time delays are conservative to some extent. To reduce the potential conservatism, we can select the following functional [5], [54]:

$$
\begin{aligned}
\hat{V}_{\sigma_{k}}(k)= & V_{\sigma_{k}}(k)-e^{T}(k) P_{\sigma_{k}} e(k)+\hat{\eta}^{T}(k) \hat{P}_{\sigma_{k}} \hat{\eta}(k) \\
& +\sum_{l=k-\underline{\tau}}^{k-1} \rho_{k l} e^{T}(l) S_{\sigma_{k}}^{1} e(l)+\sum_{l=k-\bar{\tau}}^{k-\underline{\tau}-1} \rho_{k l} e^{T}(l) S_{\sigma_{k}}^{2} e(l)
\end{aligned}
$$

$$
\begin{aligned}
& +\sum_{j=-\tau}^{-1} \sum_{l=k+j}^{k-1} \rho_{k l} y^{T}(l) R_{\sigma_{k}}^{1} y(l) \\
& +\sum_{j=-\bar{\tau}}^{-\underline{\tau}-1} \sum_{l=k+j}^{k-1} \rho_{k l} y^{T}(l) R_{\sigma_{k}}^{2} y(l) \\
& +\sum_{i=1}^{+\infty} \nu_{i} \sum_{j=1}^{i} \sum_{l=k-j}^{k-1} \rho_{k l} \bar{h}^{T}(e(l)) R_{\sigma_{k}}^{3} \bar{h}(e(l))
\end{aligned}
$$

where $V_{\sigma_{k}}(k)$ is given in (15), $P_{\alpha}>0, \hat{P}_{\alpha}>0, S_{\alpha}^{\tilde{i}}>0$, $R_{\alpha}^{\tilde{j}}>0(\alpha \in \mathcal{M}, \tilde{i}=1,2, \tilde{j}=1,2,3), \rho_{k l} \triangleq \rho^{k-l-1}, y(k) \triangleq$ $e(k+1)-e(k)$, and $\hat{\eta}(k) \triangleq\left[\begin{array}{cc}e^{T}(k) & \tilde{\eta}^{T}(k)\end{array}\right]^{T}$ with $\tilde{\eta}(k) \triangleq$ $\left[\sum_{l=k-\underline{\tau}}^{k-1} e^{T}(l) \quad \sum_{l=k-\bar{\tau}}^{k-\tau} e^{T}(l) \quad \sum_{j=1}^{+\infty} \nu_{j} \sum_{l=k-j}^{k-1} \bar{h}(e(l))\right]^{T}$.

Correspondingly, under the constraint conditions

$$
\begin{gathered}
\left|u_{i(j)}(k)-v_{i(j)}(k)-\mathcal{L}_{i(j)}^{\sigma_{k}} \tilde{\eta}(k)\right| \leq \bar{u}_{i(l)}, \\
i=1,2, \ldots, N, \quad j=1,2, \ldots, m, k \geq 0
\end{gathered}
$$

where $\mathcal{L}_{i}^{\alpha}(\alpha \in \mathcal{M})$ is any $m \times 3 n N$ constant matrix, the sector condition (14) can be updated as follows:

$$
\begin{gathered}
\psi^{T}(u(k)) T_{\sigma_{k}}\left[\psi(u(k))-U_{\sigma_{k}} e(k)-V_{\sigma_{k}} e\left(k-\tau_{k}\right)\right. \\
\left.-W_{\sigma_{k}} \sum_{l=1}^{+\infty} \nu_{l} \bar{h}(e(k-l))-\mathcal{L}_{\sigma_{k}} \tilde{\eta}(k)\right] \leq 0
\end{gathered}
$$

where $\mathcal{L}_{\sigma_{k}} \triangleq\left[\begin{array}{lll}\left(\mathcal{L}_{1}^{\sigma_{k}}\right)^{T} & \left(\mathcal{L}_{2}^{\sigma_{k}}\right)^{T} \cdots\left(\mathcal{L}_{N}^{\sigma_{k}}\right)^{T}\end{array}\right]^{T}$.

By further incorporating the recent developed inequalities [5], [53], [54], the less conservative conditions can be readily established. However, it is worth mentioning more computation burden will be involved in the obtained results.

Remark 10: When the coupling delay and the input delay are further contained in the dynamical network (1), the closedloop error dynamics (9) can be revised as follows:

$$
\begin{aligned}
& e(k+1)=\left(\bar{C}+\bar{F}_{1}\right) e(k)+\bar{G}_{1} e\left(k-\tau_{k}\right)+\bar{L}_{\sigma_{k}} e\left(k-h_{k}\right) \\
& \quad+\bar{B} K_{\sigma_{k}} e\left(k-d_{k}\right)+\bar{A} \bar{f}(e(k))+\bar{D}_{1} \bar{g}\left(e\left(k-\tau_{k}\right)\right) \\
& \quad+\bar{D}_{2} \sum_{l=1}^{+\infty} \nu_{l} \bar{h}(e(k-l))-B \psi\left(u\left(k-d_{k}\right)\right)+\bar{E} \omega(k)
\end{aligned}
$$

where $h_{k}$ and $d_{k}$ are, respectively, the coupling delay and the input delay. Note that the dynamical network (1) might be not self-synchronizing. In this case, the analysis approach proposed in this paper cannot be applicable. However, we can establish the corresponding conditions by using the techniques stated in Remark 9 to deal with the coupling and input delays.

\section{NUMERICAL EXAMPLES}

Example 1: Consider the discrete-time delayed dynamical network (1) consisting of three nodes (4), where

$$
\begin{aligned}
C & =\left[\begin{array}{cc}
-0.5 & 0.2 \\
0 & 0.95
\end{array}\right], f(x)=\left[\begin{array}{c}
\tanh \left(0.2 x_{1}\right) \\
-\tanh \left(0.75 x_{2}\right)
\end{array}\right], \\
g(x) & =h(x)=\left[\begin{array}{c}
0.2 x_{1}-\tanh \left(0.1 x_{1}\right) \\
0.1 x_{2}
\end{array}\right], \Gamma=0.4 I, \\
A & =B=D_{1}=D_{2}=E=I, \bar{u}_{i(j)}=10, \\
\tau_{k} & =3+\left[1+(-1)^{k}\right] / 2, \nu_{l}=2^{-l} .
\end{aligned}
$$


For this example, we assume that the network topology is switching among three modes. Correspondingly, the outercoupling matrices are given as follows:

$$
\begin{aligned}
L_{1} & =\left[\begin{array}{ccc}
-0.2 & 0.1 & 0.1 \\
0.1 & -0.2 & 0.1 \\
0.1 & 0.1 & -0.2
\end{array}\right], \\
L_{2} & =\left[\begin{array}{ccc}
-0.1 & 0.1 & 0 \\
0.1 & -0.2 & 0.1 \\
0 & 0.1 & -0.1
\end{array}\right], \\
L_{3} & =\left[\begin{array}{ccc}
-0.3 & 0.1 & 0.2 \\
0.1 & -0.1 & 0 \\
0.2 & 0 & -0.2
\end{array}\right] .
\end{aligned}
$$

By calculations, it is checked that $\underline{\tau}=3, \bar{\tau}=4$ and

$$
\begin{aligned}
& F_{1}=\left[\begin{array}{cc}
0 & 0 \\
0 & -0.75
\end{array}\right], F_{2}=\left[\begin{array}{cc}
0.2 & 0 \\
0 & 0
\end{array}\right], \\
& G_{1}=H_{1}=\left[\begin{array}{cc}
0.1 & 0 \\
0 & 0.1
\end{array}\right], G_{2}=H_{2}=\left[\begin{array}{cc}
0.2 & 0 \\
0 & 0.1
\end{array}\right] .
\end{aligned}
$$

First, we address the case without external disturbances. Letting $\mu=1.08, \varphi_{\alpha}=0.2, \psi_{\alpha}=\iota_{\alpha}=1(\alpha=1,2,3), \mathbb{N}_{0}=$ 1 , and solving Prob.3 and Prob.3', the maximum admissible $r$ of the initial condition set $\mathscr{X}_{r}$ can be easily obtained for different $\rho$, which are listed in Table I. In particular, when $\rho=0.98$, we have the following the controller gains:

$$
\begin{aligned}
K_{1}^{1} & =\left[\begin{array}{cc}
0.4991 & -0.1260 \\
-0.0888 & -0.4921
\end{array}\right], K_{2}^{1}=\left[\begin{array}{cc}
0.5243 & -0.1491 \\
-0.0685 & -0.5540
\end{array}\right], \\
K_{3}^{1} & =\left[\begin{array}{cc}
0.5191 & -0.1427 \\
-0.0709 & -0.5363
\end{array}\right], K_{1}^{2}=\left[\begin{array}{cc}
0.5329 & -0.1496 \\
-0.0623 & -0.3441
\end{array}\right], \\
K_{2}^{2} & =\left[\begin{array}{cc}
0.3643 & -0.1636 \\
-0.1210 & -0.6686
\end{array}\right], K_{3}^{2}=\left[\begin{array}{cc}
0.6084 & -0.1669 \\
-0.0383 & -0.4308
\end{array}\right], \\
K_{1}^{3} & =\left[\begin{array}{cc}
0.3785 & -0.1047 \\
-0.1525 & -0.5215
\end{array}\right], K_{2}^{3}=\left[\begin{array}{cc}
0.6384 & -0.1901 \\
-0.0391 & -0.4848
\end{array}\right], \\
K_{3}^{3} & =\left[\begin{array}{cc}
0.6275 & -0.1785 \\
-0.0683 & -0.5432
\end{array}\right] .
\end{aligned}
$$

Using the above controller gains, the synchronization errors are plotted in Figs. 1-2. In the simulation, the initial conditions are selected as $\phi_{1}(\theta)=\left[\begin{array}{ll}12 & 9\end{array}\right]^{T}, \phi_{2}(\theta)=\left[\begin{array}{ll}17 & 30\end{array}\right]^{T}, \phi_{3}(\theta)=$ $\left[\begin{array}{ll}22 & 48\end{array}\right]^{T}, \phi(\theta)=\left[\begin{array}{ll}15 & 25\end{array}\right]^{T}(\theta \leq 0)$. It is clear that $\bar{\phi}(\theta)=$ $\left[\begin{array}{llllll}-3 & -16 & 2 & 5 & 7 & 23\end{array}\right]^{T} \in \mathscr{X}_{r}$. The switching signal is shown in Fig. 3, where $\mathbb{T}_{a} \geq \mathbb{T}_{a}^{*}=-\ln 1.08 / \ln 0.98=3.8094$ and $\mathbb{N}_{0}=1$. It is seen from Figs. 1-2 that the synchronization can be successfully achieved under the proposed control scheme. Figs. 4-5 shows that the synchronization cannot be guaranteed without imposing the controller. On the other hand, it is seen from Table I that Prob. 3 can provide a larger estimate of the initial condition set $\mathscr{X}_{r}$ than Prob.3'. Recalling that Prob.3' is based on the delay-independent sector conditions, it can be concluded that our proposed mixed-delay-dependent sector conditions are really effective in reducing the conservatism.

Next, we will address the case that both the network and the isolate node have disturbances. By solving Prob.1 and Prob.1' with $\mu=1.08, \varphi_{\alpha}=0.2, \psi_{\alpha}=\iota_{\alpha}=1(\alpha=1,2,3)$ and $\mathbb{N}_{0}=1$, the maximum disturbance tolerance levels $\delta_{M}$ are obtained for different $\rho$, which are given in Table II. From
Table II, it is seen that Prob.1 provides the larger disturbance tolerance levels $\bar{\delta}_{M}$ than Prob.1', which verifies again that our proposed mixed-delay-dependent sector conditions are more effective. When $\rho=0.98$, letting $\delta=200 \leq \delta_{M}$ and solving Prob.2 with $\mu=1.08, \varphi_{\alpha}=0.3, \psi_{\alpha}=\iota_{\alpha}=1(\alpha=1,2,3)$ and $\mathbb{N}_{0}=1$, we obtain the minimum $l_{2}-l_{\infty}$ performance level $\gamma^{*}=3.1836$ and the following controller gains:

$$
\begin{aligned}
K_{1}^{1} & =\left[\begin{array}{cc}
0.4734 & -0.1903 \\
-0.0190 & -0.5114
\end{array}\right], K_{2}^{1}=\left[\begin{array}{cc}
0.4742 & -0.1977 \\
-0.0052 & -0.5095
\end{array}\right], \\
K_{3}^{1} & =\left[\begin{array}{cc}
0.4743 & -0.2007 \\
-0.0031 & -0.5098
\end{array}\right], K_{1}^{2}=\left[\begin{array}{cc}
0.4447 & -0.1943 \\
-0.0127 & -0.5390
\end{array}\right], \\
K_{2}^{2} & =\left[\begin{array}{cc}
0.4818 & -0.1976 \\
-0.0043 & -0.4980
\end{array}\right], K_{3}^{2}=\left[\begin{array}{cc}
0.4445 & -0.1959 \\
-0.0080 & -0.5374
\end{array}\right], \\
K_{1}^{3} & =\left[\begin{array}{cc}
0.5040 & -0.1787 \\
-0.0235 & -0.4522
\end{array}\right], K_{2}^{3}=\left[\begin{array}{cc}
0.4664 & -0.2101 \\
0.0194 & -0.5318
\end{array}\right], \\
K_{3}^{3} & =\left[\begin{array}{ll}
0.5068 & -0.2149 \\
0.0251 & -0.4920
\end{array}\right],
\end{aligned}
$$

Choose the external disturbances as follows:

$$
\begin{aligned}
& \omega_{1}(k)=\left[\begin{array}{ll}
4.5 & 4.5
\end{array}\right]^{T}, \omega_{2}(k)=\left[\begin{array}{ll}
3.6 & 0
\end{array}\right]^{T}, 0 \leq k \leq 4, \\
& \omega_{3}(k)=\left[\begin{array}{ll}
3 & -0.6
\end{array}\right]^{T}, \omega(k)=\left[\begin{array}{ll}
1.5 & 3
\end{array}\right]^{T}, 0 \leq k \leq 4, \\
& \omega_{1}(k)=\omega_{2}(k)=\omega_{3}(k)=\omega(k)=\left[\begin{array}{ll}
0 & 0
\end{array}\right]^{T}, k \geq 5 .
\end{aligned}
$$

Then, it follows that $\bar{\omega}(k)=\left[\begin{array}{llllll}3 & 1.5 & 2.1 & -3 & 1.5 & -3.6\end{array}\right]^{T}$ $(0 \leq k \leq 4), \bar{\omega}(k)=\left[\begin{array}{llllll}0 & 0 & 0 & 0 & 0 & 0\end{array}\right]^{T}(k \geq 5)$. It is checked that $\sum_{k=0}^{+\infty} \bar{\omega}^{T}(k) \bar{\omega}(k)=199.35 \leq 200$. Using above controller gains and disturbances, and the switching signal in Fig. 3, the synchronization errors are plotted in Figs. 6-7 and the truncated $l_{2}-l_{\infty}$ gain $\gamma_{k}$ is shown in Fig. 8 under zero initial conditions $\left(\phi_{1}=\phi_{2}=\phi_{3}=\phi=0\right)$. In the simulation, the truncated $l_{2}-l_{\infty}$ gain $\gamma_{k}$ is defined as $\gamma_{k}=\left[\sup _{0 \leq i \leq k} e^{T}(i) e(i)\right] /\left[\sum_{i=0}^{k} \bar{\omega}^{T}(i) \bar{\omega}(i)\right]$. From Figs. 6-8, it is obvious that the synchronization can be achieved well and the truncated $l_{2}-l_{\infty}$ gain $\gamma_{k}$ is less than $\gamma^{*}=3.1836$.

Example 2: Let us consider a coupled neural network with mixed delays [36], [49], which can be described by the model (1) with the following parameters:

$$
\begin{aligned}
C & =\left[\begin{array}{cc}
0.9 & 0 \\
0 & 0.9
\end{array}\right], A=\left[\begin{array}{cc}
0.18 & -0.015 \\
-0.52 & 0.35
\end{array}\right], \\
D_{1} & =\left[\begin{array}{cc}
-0.17 & -0.012 \\
-0.026 & -0.25
\end{array}\right], D_{2}=\left[\begin{array}{cc}
0.06 & 0.015 \\
-0.2 & -0.01
\end{array}\right], \\
B & =I, E=0, \Gamma=0.3 I, N=3, \bar{u}_{i(j)}=15, \\
f(x) & =g(x)=h(x)=\left[\tanh \left(x_{1}\right) \quad \tanh \left(x_{2}\right)\right]^{T}, \\
\tau_{k} & =9+\left[1+(-1)^{k}\right] / 2, \nu_{l}=e^{-0.5 l} / 2 .
\end{aligned}
$$

Here, the network topology is assumed to be switching among three modes, where the outer-coupling matrices $L_{1}$ and $L_{2}$ are the same as in Example 1 and $L_{3}$ is given as follows:

$$
L_{3}=\left[\begin{array}{ccc}
-0.1 & 0 & 0.1 \\
0 & -0.1 & 0.1 \\
0.1 & 0.1 & -0.2
\end{array}\right] \text {. }
$$

It is seen that $\underline{\tau}=9, \bar{\tau}=10$ and the nonlinear functions $f(x)=g(x)=h(x)$ satisfy the conditions in (3) with

$$
F_{1}=G_{1}=H_{1}=0, F_{2}=G_{2}=H_{2}=I .
$$




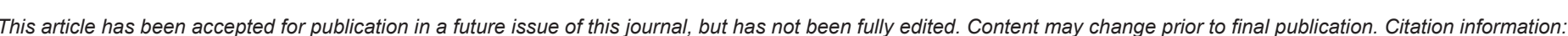
DOI10.1109/TNNLS.2020.2996094, IEEE Transactions on Neural Networks and Learning Systems

TABLE I

MAXIMUM ADMISSIBLE $r$ OF THE SET $\mathscr{X}_{r}$ FOR DIFFERENT $\rho$

\begin{tabular}{cccccc}
\hline$\rho$ & 0.95 & 0.96 & 0.97 & 0.98 & 0.99 \\
\hline Prob.3 $^{\prime}$ & 26.0860 & 27.2126 & 28.3983 & 29.6533 & 30.9877 \\
\hline Prob. $^{\prime}$ & 21.5334 & 22.5339 & 23.5960 & 24.7262 & 25.9317 \\
\hline Improvements & $21.14 \%$ & $20.76 \%$ & $20.35 \%$ & $19.93 \%$ & $19.50 \%$ \\
\hline
\end{tabular}

TABLE II

MAXIMUM DISTURBANCE TOLERANCE LEVELS $\delta_{M}$ FOR DIFFERENT $\rho$

\begin{tabular}{cccccc}
\hline$\rho$ & 0.95 & 0.96 & 0.97 & 0.98 & 0.99 \\
\hline Prob.1 & 179.862 & 193.601 & 207.011 & 220.271 & 233.618 \\
\hline Prob.1 & 131.432 & 142.586 & 153.431 & 164.094 & 174.796 \\
\hline Improvements & $36.85 \%$ & $35.78 \%$ & $34.92 \%$ & $34.23 \%$ & $33.65 \%$ \\
\hline
\end{tabular}

For this example, Fig. 9 shows that the chaotic behavior occurs for the unforced isolate node with the initial condition $\phi(\theta)=\left[\begin{array}{ll}0.4 & 0.5\end{array}\right]^{T}(\theta \leq 0)$. Moreover, it can be verified that the open-loop error dynamics is not stable, which means that the synchronization cannot be ensured for the coupled neural network without using the controller. Choosing $\mu=1.06, \rho=$ $0.98, \psi_{\alpha}=\iota_{\alpha}=1(\alpha=1,2,3), \mathbb{N}_{0}=1$, and solving Prob.3, one obtains the maximum admissible bound $r=9.5711$ of the initial condition set $\mathscr{X}_{r}$ and the following controller gains:

$$
\begin{aligned}
& K_{1}^{1}=\left[\begin{array}{cc}
-0.9937 & 0.0915 \\
0.2513 & -1.0058
\end{array}\right], \\
& K_{2}^{1}=K_{3}^{1}=\left[\begin{array}{cc}
-1.0016 & 0.0821 \\
0.2524 & -1.0088
\end{array}\right], \\
& K_{1}^{2}=K_{1}^{3}=\left[\begin{array}{cc}
-0.9720 & 0.1098 \\
0.2555 & -1.0239
\end{array}\right], \\
& K_{2}^{2}=K_{3}^{3}=\left[\begin{array}{cc}
-1.1125 & -0.0104 \\
0.2529 & -1.0135
\end{array}\right], \\
& K_{3}^{2}=K_{2}^{3}=\left[\begin{array}{cc}
-0.9717 & 0.1099 \\
0.2556 & -1.0239
\end{array}\right] .
\end{aligned}
$$

Choosing $\phi_{1}(\theta)=\left[\begin{array}{ll}3.4 & -4.5\end{array}\right]^{T}, \phi_{2}(\theta)=\left[\begin{array}{ll}2.4 & -2.5\end{array}\right]^{T}$, $\phi_{3}(\theta)=\left[\begin{array}{ll}-1.6 & 6.5\end{array}\right]^{T}, \phi(\theta)=\left[\begin{array}{cc}0.4 & 0.5\end{array}\right]^{T}, \theta \in(-\infty, 0]$, we have $\bar{\phi}(\theta)=\left[\begin{array}{llllll}3 & -5 & 2 & -3 & -2 & 6\end{array}\right]^{T} \in \mathscr{X}_{r}$. Using the above controller gains and initial conditions, the state evolutions of the controlled coupled neural network and the unforced isolate neural network are plotted Figs. 10-11. In the simulation, the switching signal is given in Fig. 12, where $\mathbb{T}_{a} \geq \mathbb{T}_{a}^{*}=-\ln 1.06 / \ln 0.98=2.8842$. It is seen from Figs. 10-11 that our proposed control scheme performs well.

\section{CONCLUSions}

In this paper, we have addressed the synchronization control problem for a class of discrete-time delayed dynamical networks with switching topology under actuator saturations. To alleviate the saturation effect effectively, novel mixed-delaydependent sector conditions have been proposed. By further incorporating the piecewise Lyapunov-like functional and the ADT switching, a sufficient condition has been obtained under which the error dynamics has some desirable features including the boundedness of error states, $l_{2}-l_{\infty}$ performance and

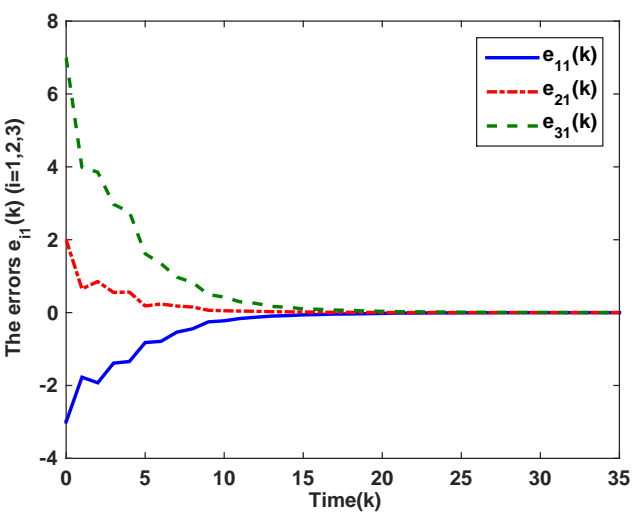

Fig. 1. The state evolutions of the controlled error dynamics.

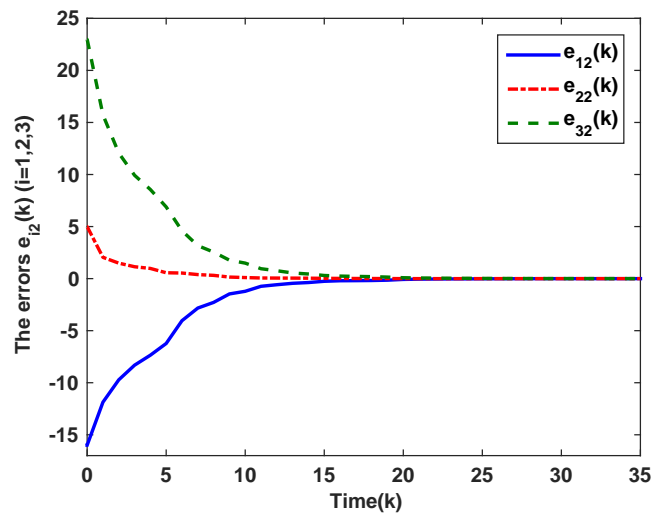

Fig. 2. The state evolutions of the controlled error dynamics.

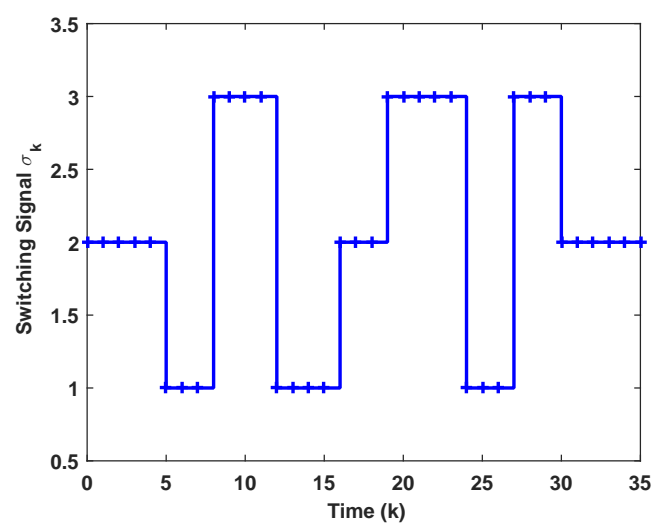

Fig. 3. The switching signal $\sigma_{k}$ of the network topology.

the exponential stability. Then, the explicit characterization of controller gains has been proposed by solving of a set of LMIs. Subsequently, three corresponding optimization problems have been proposed. Finally, two examples have been given to illustrate the feasibility and benefits of our obtained results.

On the other hand, it is worth mentioning that the technique of analyzing the switched dynamics in this paper is somewhat conservative. As the further work, we would like 
This article has been accepted for publication in a future issue of this journal, but has not been fully edited. Content may change prior to final publication. Citation information: DOI10.1109/TNNLS.2020.2996094, IEEE Transactions on Neural Networks and Learning Systems

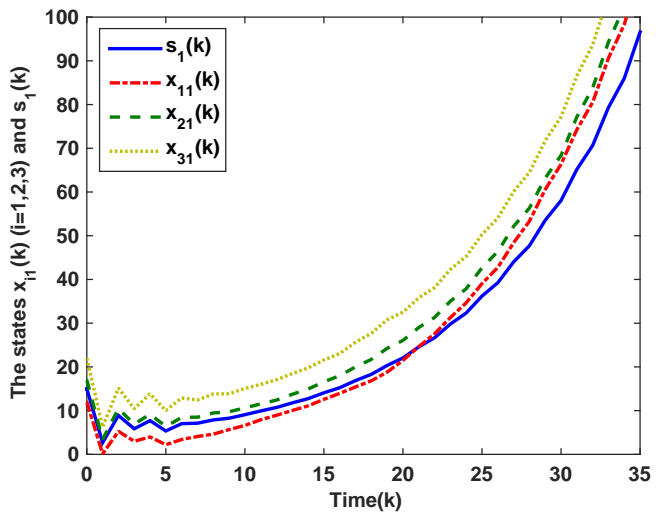

Fig. 4. The state evolutions of the uncontrolled dynamical network and the unforced isolate node.

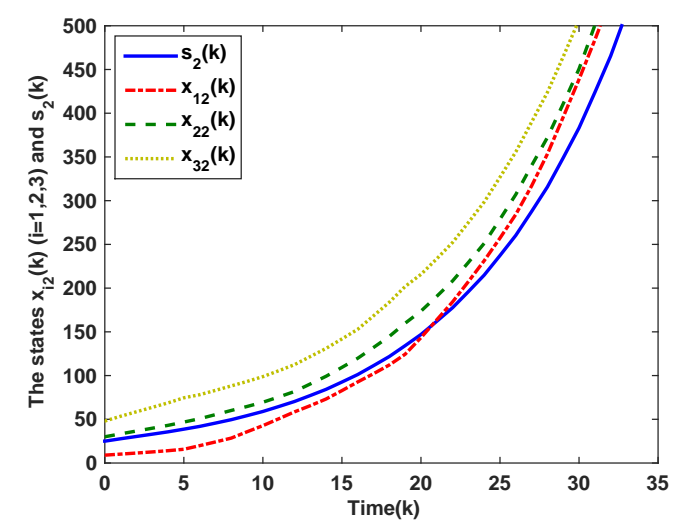

Fig. 5. The state evolutions of the uncontrolled dynamical network and the unforced isolate node.

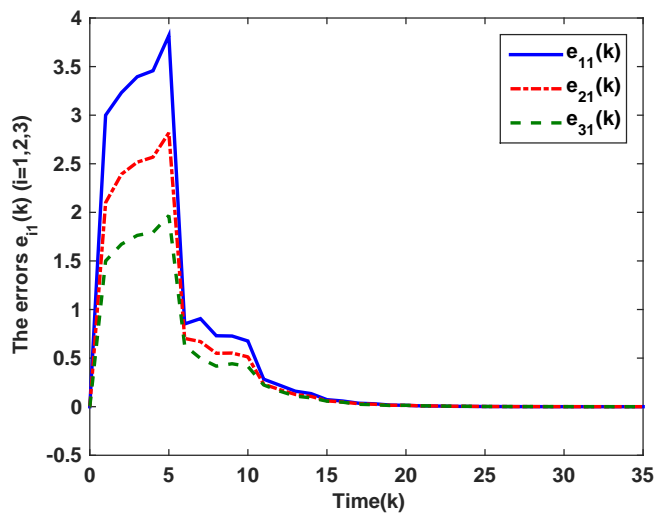

Fig. 6. The state evolutions of the controlled error dynamics.

to establish some more effective results by incorporating the mode-dependent ADT switching [60] and the persistent dwelltime switching [55]. Also, it is more interesting to design the state-dependent switching rules to ensure the synchronization of dynamical networks [59]. In fact, we can establish the corresponding synchronization criteria by designing the state-

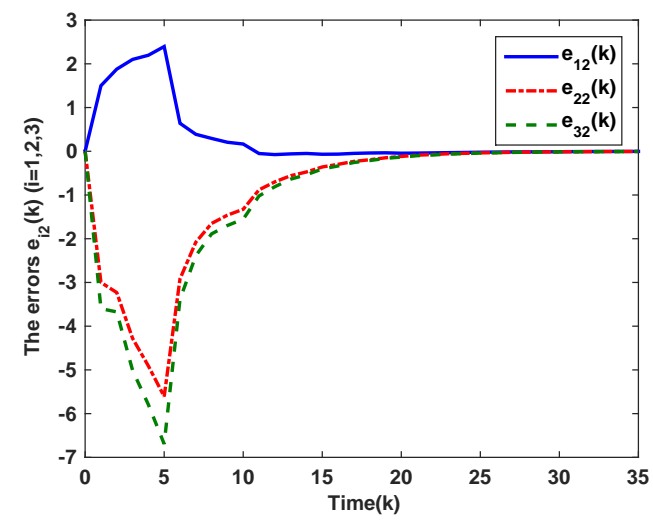

Fig. 7. The state evolutions of the controlled error dynamics.

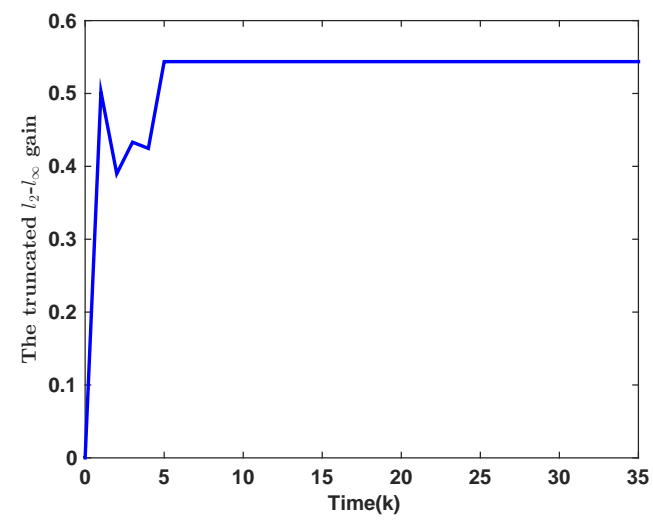

Fig. 8. The truncated $l_{2}-l_{\infty}$ gain $\gamma_{k}$ of the error dynamics.

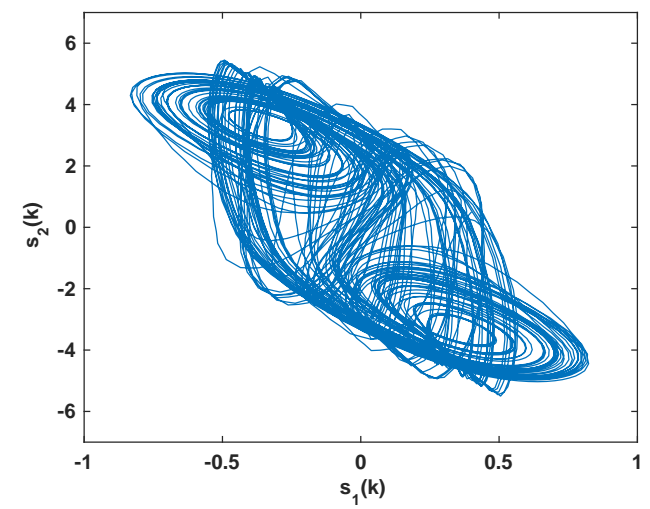

Fig. 9. Chaotic behavior of the unforced isolate node.

dependent switching law $\sigma(k)=\operatorname{argmin}\left\{V_{\alpha}(k), \alpha \in \mathcal{M}\right\}$ [57]. In addition, it should be pointed out that our proposed results can be easily extended to the case that the controller switching and the topology switching are not consistent.

\section{REFERENCES}

[1] S. Boccaletti, V. Latora, Y. Moreno, M. Chavez, and D.-U. Hwang, "Complex networks: Structure and dynamics", Phys. Rep., vol. 424, no. 4-5, 175-308, Feb. 2006. 


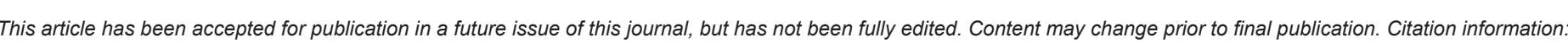
DOI10.1109/TNNLS.2020.2996094, IEEE Transactions on Neural Networks and Learning Systems

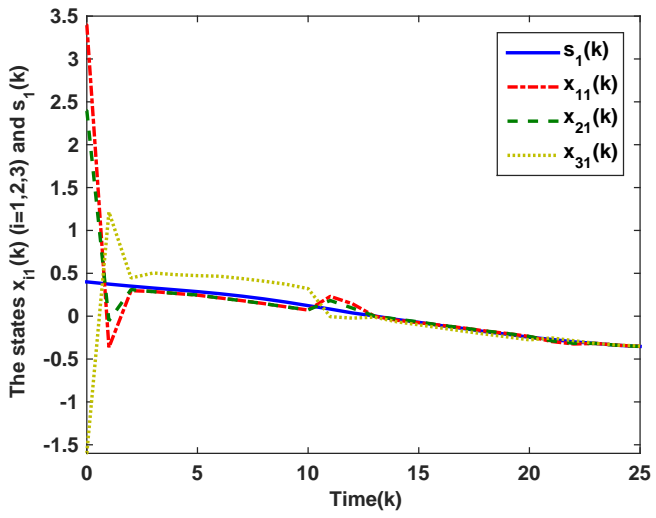

Fig. 10. The state evolutions of the controlled dynamical network and the unforced isolate node.

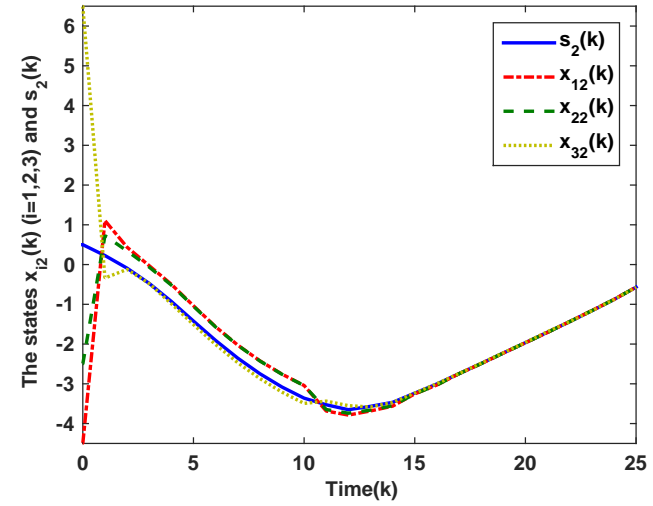

Fig. 11. The state evolutions of the controlled dynamical network and the unforced isolate node.

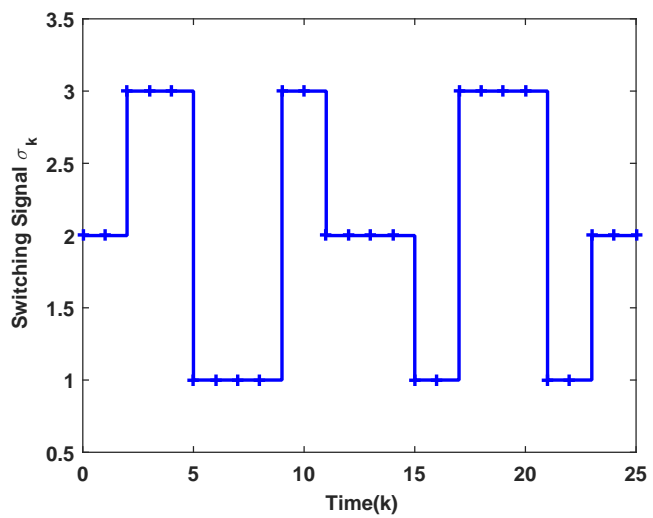

Fig. 12. The switching signal $\sigma_{k}$ of the network topology.

[2] S. Cai, J. Hao, Q. He, and Z. Liu, "Exponential synchronization of complex delayed dynamical networks via pinning periodically intermittent control", Phys. Lett. A, vol. 375, no. 19, pp. 1965-1971, May 2011.

[3] M. Z. Q. Chen, L. Zhang, H. Su, and G. Chen, "Stabilizing solution and parameter dependence of modified algebraic Riccati equation with application to discrete-time network synchronization", IEEE Trans. Autom. Control, vol. 61, no. 1, pp. 228-233, Jan. 2016.

[4] Y. Chen, S. Fei, and Y. Li, "Robust stabilization for uncertain saturated time-delay systems: a distributed-delay-dependent polytopic approach", IEEE Trans. Autom. Control, vol. 62, no. 7, pp. 3455-3460, Jul. 2017.

[5] Y. Chen, Z. Wang, Y. Liu, and F. E. Alsaadi, "Stochastic stability for distributed delay neural networks via augmented Lyapunov-Krasovskii functionals", Appl. Math. Comput., vol. 338, pp. 869-881, Dec. 2018.

[6] Y. Chen, Z. Wang, S. Fei, and Q.-L. Han, "Regional stabilization for discrete time-delay systems with actuator saturations via a delaydependent polytopic approach", IEEE Trans. Autom. Control, vol. 64, no. 3, pp. 1257-1264, Mar. 2019.

[7] Y. Chen, Z. Wang, B. Shen, and H. Dong, "Exponential synchronization for delayed dynamical networks via intermittent control: dealing with actuator saturations", IEEE Trans. Neural Netw. Learn. Syst., vol. 30, no. 4, pp. 1000-1013, Apr. 2019.

[8] H. D. Choi, C. K. Ahn, H. R. Karimi and M. T. Lim, "Filtering of discrete-time switched neural networks ensuring exponential dissipative and $l_{2}-l_{\infty}$ performances", IEEE Trans. Cybern., vol. 47, no. 10, pp. 3195-3207, Oct. 2017.

[9] P. DeLellis, M. di Bernardo, and F. Garofalo, "Adaptive pinning control of networks of circuits and systems in Lur'e form", IEEE Trans. Circuits Syst. I, Reg. Papers, vol. 60, no. 11, pp. 3033-3042, Nov. 2013.

[10] L. Hou, G. Zong, Y. Wu, and Y. Cao, "Exponential $l_{2}-l_{\infty}$ output tracking control for discrete-time switched system with time-varying delay", Int. J. Robust Nonlinear Control, vol. 22, no. 11, pp. 1175-1194, Jul. 2012.

[11] L. Ma, Z. Wang, and H.-K. Lam, "Event-triggered mean-square consensus control for time-varying stochastic multi-agent system with sensor saturations", IEEE Trans. Autom. Control, vol. 62, no. 7, pp. 3524-3531, Jul. 2017.

[12] L. Ma, Z. Wang, Y. Liu, and F. E. Alsaadi, "A note on guaranteed cost control for nonlinear stochastic systems with input saturation and mixed time-delays", Int. J. Robust Nonlinear Control, vol. 27, no. 18 , pp. 4443-4456, Dec. 2017.

[13] C. Li, and G. Chen, "Synchronization in general complex dynamical networks with coupling delays", Physica A, vol. 343, 263-278, Nov. 2004.

[14] Q. Li, B. Shen, Z. Wang, T. Huang, and J. Luo, "Synchronization control for a class of discrete time-delay complex dynamical networks: A dynamic event-triggered approach", IEEE Trans. Cybern., vol. 49, no. 5, pp. 1979-1986, May 2019,

[15] C. Li, W. Yu, and T. Huang, "Impulsive synchronization schemes of stochastic complex networks with switching topology: Average time approach", Neural Netw., vol. 54, pp. 85-94, Jun. 2014.

[16] Q. Li, B. Shen, Z. Wang, T. Huang and J. Luo, Synchronization control for a class of discrete time-delay complex dynamical networks: a dynamic event-triggered approach, IEEE Transactions on Cybernetics, vol. 49, no. 5, pp. 1979-1986, May 2019.

[17] K. Liang, M. Dai, H. Shen, et al., " $L_{2}-L_{\infty}$ synchronization for singularly perturbed complex networks with semi-Markov jump topology", Appl. Math. Comput., vol. 321, pp. 450-462, Mar. 2018.

[18] D. Liberzon, Switching in Systems and Control. Boston, MA, USA: Birkhäuser, 2003.

[19] Y. Li, Z. Lin, Stability and Performance of Control Systems with Actuator Saturation. Basel, Switzerland: Birkhäuser, 2018.

[20] Z. Lin, Low Gain Feedback. London, U.K.: Springer-Verlag, 1999.

[21] X. Liu, and T. Chen, "Synchronization of linearly coupled networks with delays via aperiodically intermittent pinning control", IEEE Trans. Neural Netw. Learn. Syst., vol. 26, no. 10, pp. 2396-2407, Oct. 2015.

[22] B. Liu, W. Lu, and T. Chen, "Synchronization in complex networks with stochastically switching coupling structures", IEEE Trans. Autom. Control, vol. 57, no. 3, pp. 754-760, Mar. 2012.

[23] Y. Liu, Z. Wang, J. Liang, and X. Liu, "Synchronization and state estimation for discrete-time complex networks with distributed delays", IEEE Trans. Syst., Man, Cybern. B, Cybern., vol. 38, no. 5, pp. 1314 1325, Oct. 2008.

[24] Y. Liu, Z. Wang, L. Ma, Y. Cui, and F. E. Alsaadid, "Synchronization of directed switched complex networks with stochastic link perturbations and mixed time-delays", Nonlinear Anal.-Hybrid Syst., vol. 27, pp. 213224, Feb. 2018

[25] T. Liu, J. Zhao, and D. J. Hill, "Exponential synchronization of complex delayed dynamical networks with switching topology", IEEE Trans. Circuits Syst. I, Reg. Papers, vol. 57, no. 11, pp. 2967-2980, Nov. 2010.

[26] J. Lu, Z. Wang, J. Cao, and D. W. C. Ho, and J. Kurths, "Pinning impulsive stabilization of nonlinear dynamical networks with timevarying delay", Int. J. Bifurcation Chaos, vol. 22, no. 7, 1250176, 2012.

[27] W. Lu, T. Chen, "Synchronization of coupled connected neural networks with delays", IEEE Trans. Circuits Syst. II, Exp. Briefs, vol. 51, no. 12, pp.2491-2503, Dec. 2004. 
This article has been accepted for publication in a future issue of this journal, but has not been fully edited. Content may change prior to final publication. Citation information: DOI10.1109/TNNLS.2020.2996094, IEEE Transactions on Neural Networks and Learning Systems

[28] W. Lu, T. Chen, "New approach to synchronization analysis of linearly coupled ordinary differential systems", Physica D, vol. 213, no. 2, pp. 214-230, Jan. 2006.

[29] W. Qian, L. Wang, M. Z. Q. Chen, "Local consensus of nonlinear multiagent systems with varying delay coupling", IEEE Trans. Syst., Man, Cybern., Syst., vol. 48, no. 12, pp. 2462-2469, Dec. 2018

[30] B. Shen, Z. Wang, and X. Liu, "Sampled-data synchronization control of dynamical networks with stochastic sampling", IEEE Trans. Autom. Control, vol. 57, no. 10, pp. 2644-2650, Oct. 2012.

[31] B. Shen, Z. Wang, D. Wang and Q. Li, State-saturated recursive filter design for stochastic time-varying nonlinear complex networks under deception attacks, IEEE Transactions on Neural Networks and Learning Systems, in press, DOI: 10.1109/TNNLS.2019.2946290.

[32] O. Solomon, and E. Fridman, "New stability conditions for systems with distributed delays", Automatica, vol. 49, no. 11, pp. 3467-3475, Nov. 2013.

[33] G. Song, J. Lam, and S. Xu, "Quantized feedback stabilization of continuous time-delay systems subject to actuator saturation", Nonlinear Anal.-Hybrid Syst., vol. 30, pp. 1-13, Nov. 2018.

[34] D. J. Stilwell, E. M. Bollt, and D. G. Roberson, "Sufficient conditions for fast switching synchronization in time-varying network topologies", SIAM J. Appl. Dyn. Syst., vol. 5, no. 1, pp. 140-156, 2006.

[35] J. Suo, Z. Wang and B. Shen, Pinning synchronization control for a class of discrete-time switched stochastic complex networks under eventtriggered mechanism, Nonlinear Analysis: Hybrid Systems, Vol. 37, Aug. 2020, Art. No. 100886, 15 pages.

[36] Y. Tang, J.-A. Fang, and Q.-Y. Miao, "Synchronization of stochastic delayed neural networks with Markovian switching and its application", Int. J. Neural Syst., vol. 19, no. 1, pp. 43-56, 2009.

[37] S. Tarbouriech, G. Garcia, J. M. G. da Silva, and I. Queinnec, Stability and Stabilization of Linear Systems with Saturating Actuators. London, U.K.: Springer-Verlag, 2011.

[38] X. Wan, Z. Wang, M. Wu, and X. Liu, " $H_{\infty}$ state estimation for discretetime nonlinear singularly perturbed complex networks under the RoundRobin Protocol", IEEE Trans. Neural Netw. Learn. Syst., vol. 30, no. 2 , pp. 415-426, Feb. 2019

[39] J.-L. Wang, Z. Qin, H.-N. Wu, and T. Huang, Finite-time synchronization and $H_{\infty}$ synchronization of multiweighted complex networks with adaptive state couplings", IEEE Trans. Cybern., to be published, doi: 10.1109/TCYB.2018.2870133.

[40] L. Wang, Z. Wang, Q.-L. Han, and G. Wei, "Synchronization control for a class of discrete-time dynamical networks with packet dropouts: A coding-decoding-based approach", IEEE Trans. Cybern., vol. 48, no. 8, pp. 2437-2448, Apr. 2018.

[41] L. Wang, Z. Wang, T. Huang, and G. Wei, "An event-triggered approach to state estimation for a class of complex networks with mixed time delays and nonlinearities", IEEE Trans. Cybern., vol. 46, no. 11, pp. 2497-2508, Nov. 2016.

[42] Q. Wang, Z.-G. Wu, P. Shi, H. Yan, and Y. Chen, "Stability analysis and control for switched system with bounded actuators", IEEE Trans. Syst., Man, Cybern., Syst., to be published, doi: 10.1109/TSMC.2018.2854282.

[43] X. F. Wang, "Complex networks: topology, dynamics and synchronization", Int. J. Bifurcation Chaos, vol. 12, no. 5, pp. 885-916, May 2002.

[44] Z. Wang, Y. Wang, and Y. Liu, "Global synchronization for discrete-time stochastic complex networks with randomly occurred nonlinearities and mixed time delays", IEEE Trans. Neural Netw., vol. 21, no. 1, pp. 11-25, Jan. 2010.

[45] G. Wen, W. Yu, G. Hu, J. Cao, and X. Yu, "Pinning synchronization of directed networks with switching topologies: A multiple Lyapunoy functions approach", IEEE Trans. Neural Netw. Learn. Syst., vol. 26, no. 12, pp. 3239-3250, Dec. 2015.

[46] Y.-Q. Wu, H. Su, and Z.-G. Wu, "Synchronisation control of dynamical networks subject to variable sampling and actuators saturation", IET Control Theory Appl., vol. 9, no. 3, pp. 381-391, Feb. 2015.

[47] Y. Xie, Z. Lin, "Event-triggered global stabilization of general linear systems with bounded controls", Automatica, vol. 107, pp. 241-254, Sept. 2019.

[48] T. Yang, A. A. Stoorvogel, H. F. Grip, and A. Saberi, "Semi-global regulation of output synchronization for heterogeneous networks of nonintrospective, invertible agents subject to actuator saturation", Int. J. Robust Nonlinear Control, vol. 24, no. 3, pp. 548-566, Feb. 2014.

[49] X. Yang,Y. Feng, K. F. C. Yiu, Q. Song, and F. E. Alsaadi, "Synchronization of coupled neural networks with infinite-time distributed delays via quantized intermittent pinning control”, Nonlinear Dyn., vol. 94, no. 3, pp. 2289-2303, Nov. 2018.
[50] W. Yu, G. Chen, J. Lü, and J. Kurths, "Synchronization via pinning control on general complex networks", SIAM J. Control Optim., vol. 51, no. 2, pp. 1395-1416, Apr. 2013.

[51] H.-B. Zeng, K. L. Teo, Y. He, H. Xu, and W. Wang, "Sampled-data synchronization control for chaotic neural networks subject to actuator saturation", Neurocomputing, vol. 260, pp. 25-31, Oct. 2017.

[52] H.-B. Zeng, K. L. Teo, Y. He, and W. Wang, "Sampled-data stabilization of chaotic systems based on a T-S fuzzy model", Inf. Sci., vol. 483, pp. 262-272, May 2019.

[53] H.-B. Zeng, X.-G. Liu, and W. Wang, "A generalized free-matrix-based integral inequality for stability analysis of time-varying delay systems", Appl. Math. Comput., vol. 354, pp. 1-8, Aug. 2019.

[54] C.-K. Zhang, Y. He, L. Jiang, M. Wu, and H.-B. Zeng, "Delay-variationdependent stability of delayed discrete-time systems", IEEE Trans. Autom. Control, vol. 61, no. 9, pp. 2663-2669, Sept. 2016.

[55] L. Zhang, S. Zhuang, and P. Shi, "Non-weighted quasi-time-dependent $H_{\infty}$ filtering for switched linear systems with persistent dwell-time", Automatica, vol. 54, pp. 201-209, Apr. 2015.

[56] Q. Zhang, J. Lu, J. Lü, and C. K. Tse, "Adaptive feedback synchronization of a general complex dynamical network with delayed nodes", IEEE Trans. Circuits Syst. II, Exp. Briefs, vol. 55, no. 2, pp. 183-187, Feb. 2008

[57] X. Zhang, J. Zhao, and G. M. Dimirovski, " $L_{2}$-gain analysis and control synthesis of uncertain discrete-time switched linear systems with time delay and actuator saturation", Int. J. Control, vol. 84, no. 10, pp. 17461758, Oct. 2011.

[58] Z.-M. Zhang, Y. He, M. Wu, and Q.-G. Wang, "Exponential synchronization of neural networks with time-varying delays via dynamic intermittent output feedback control", IEEE Trans. Syst., Man, Cybern. Syst., vol. 49, no. 3, pp. 612-622, Mar. 2019.

[59] J. Zhao, D. J. Hill, and T. Liu, "Synchronization of complex dynamical networks with switching topology: A switched system point of view", Automatica, vol. 45, no. 11, pp. 2502-2511, Nov. 2009.

[60] X. Zhao, L. Zhang, P. Shi, and M. Liu. "Stability and stabilization of switched linear systems with mode-dependent average dwell time", IEEE Trans. Autom. Control, vol. 57, no. 7, pp. 1809-1815, Jul. 2012.

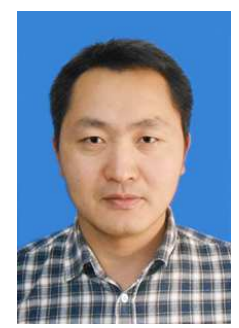

Yonggang Chen received his B.Sc. and M.Sc. degrees in Mathematics from Henan Normal University, Xinxiang, China, in 2003 and 2006, respectively. He received his Ph.D. degree in Control Theory and Control Engineering from Southeast University, Nanjing, China, in 2013.

From Apr. 2016 to Apr. 2017, he was a Visiting Scholar with the Department of Computer Science, Brunel University London, London, U.K. He is currently an Associate Professor with the School of Electrical Engineering and Automation, Henan Polytechnic University, Jiaozuo 454000, China, and is also with the School of Mathematical Sciences, Henan Institute of Science and Technology, Xinxiang, China. His research interests include time-delay systems, constrained control, neural networks, and switched system control. 


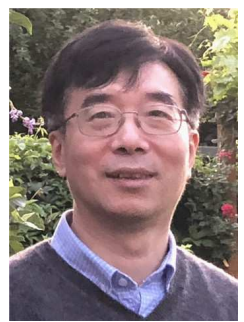

Zidong Wang (SM'03-F'14) was born in Jiangsu, China, in 1966. He received the B.Sc. degree in mathematics in 1986 from Suzhou University, Suzhou, China, and the M.Sc. degree in applied mathematics in 1990 and the Ph.D. degree in electrical engineering in 1994, both from Nanjing University of Science and Technology, Nanjing, China.

$\mathrm{He}$ is currently Professor of Dynamical Systems and Computing in the Department of Computer Science, Brunel University London, U.K. From 1990 to 2002, he held teaching and research appointments in universities in China, Germany and the UK. Prof. Wang's research interests include dynamical systems, signal processing, bioinformatics, control theory and applications. He has published around 220 papers in IEEE Transactions and around 60 papers in Automatica. He is a holder of the Alexander von Humboldt Research Fellowship of Germany, the JSPS Research Fellowship of Japan, William Mong Visiting Research Fellowship of Hong Kong.

Prof. Wang serves (or has served) as the Editor-in-Chief for Neurocomputing, the Deputy Editor-in-Chief for International Journal of Systems Science, and an Associate Editor for 12 international journals including IEEE Transactions on Automatic Control, IEEE Transactions on Control Systems Technology, IEEE Transactions on Neural Networks, IEEE Transactions on Signal Processing, and IEEE Transactions on Systems, Man, and CyberneticsPart C. He is a Fellow of the IEEE, a Fellow of the Royal Statistical Society and a member of program committee for many international conferences.

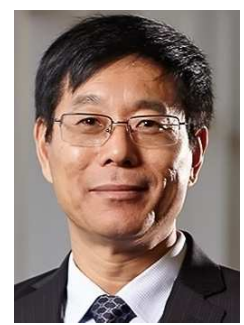

Qing-Long Han (M'09-SM'13-F'19) received the B.Sc. degree in Mathematics from Shandong Normal University, Jinan, China, in 1983, and the M.Sc. and $\mathrm{Ph} . \mathrm{D}$. degrees in Control Engineering and Electrical Engineering from East China University of Science and Technology, Shanghai, China, in 1992 and 1997, respectively.

From 1997 to 1998, he was a Post-doctoral Researcher Fellow with the Laboratoire d'Automatique et d'Informatique Industielle (currently, Laboratoire d'Informatique et d'Automatique pour les Systémes), École Supérieure d'Ing'enieurs de Poitiers (currently, École Nationale Supérieure d'Ingénieurs de Poitiers), Université de Poitiers, France. From 1999 to 2001, he was a Research Assistant Professor with the Department of Mechanical and Industrial Engineering, Southern Illinois University at Edwardsville, Edwardsville, IL, USA. From 2001 to 2014, he was a Laureate Professor, an Associate Dean of research and innovation with the Higher Education Division, and the Founding Director of the Centre for Intelligent and Networked Systems, Central Queensland University, Rockhampton, QLD, Australia. From 2014 to 2016, he was a Deputy Dean of research with the Griffith Sciences and a Professor with the Griffith School of Engineering, Griffith University, Mount Gravatt, QLD, Australia. In 2016, he joined the Swinburne University of Technology, Melbourne, VIC, Australia, where he is currently a Pro Vice-Chancellor of research quality and a Distinguished Professor. His research interests include networked control systems, multiagent systems, time-delay systems, complex dynamical systems, and neural networks.

Professor Han is a Highly Cited Researcher according to Clarivate Analytics (formerly, Thomson Reuters). He is an Associate Editor of several international journals, including the IEEE TRANSACTIONS ON CYBERNETICS, the IEEE TRANSACTIONS ON INDUSTRIAL ELECTRONICS, the IEEE TRANSACTIONS ON INDUSTRIAL INFORMATICS, IEEE Industrial Electronics Magazine, the IEEE/CAA JOURNAL OF AUTOMATICA SINICA, Control Engineering Practice, and Information Sciences. He is a fellow of the Institution of Engineers Australia.

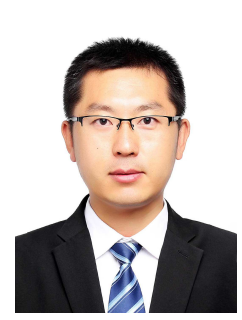

Jun Hu (M'14) received the B.Sc. degree in information and computation science and M.Sc. degree in applied mathematics from Harbin University of Science and Technology, Harbin, China, in 2006 and 2009, respectively, and the Ph.D. degree in control science and engineering from Harbin Institute of Technology, Harbin, China, in 2013.

From September 2010 to September 2012, he was a Visiting Ph.D. Student in the Department of Information Systems and Computing, Brunel University, U.K. From May 2014 to April 2016, he was an Alexander von Humboldt research fellow at the University of Kaiserslautern, Kaiserslautern, Germany. He is currently with the Department of Mathematics, Harbin University of Science and Technology, Harbin 150080, China, and also with the School of Engineering, University of South Wales, Pontypridd CF37 1DL, United Kingdom. His research interests include nonlinear control, filtering and fault estimation, time-varying systems and complex networks. $\mathrm{He}$ has published more than 50 papers in refereed international journals.

Dr. Hu serves as a reviewer for Mathematical Reviews, as an editor for Neurocomputing, Journal of Intelligent and Fuzzy Systems, Neural Processing Letters, Systems Science and Control Engineering, and as a guest editor for International Journal of General Systems and Information Fusion. 